\title{
Macrophage microRNA-150 promotes pathological angiogenesis as seen in age-related macular degeneration
}

\author{
Jonathan B. Lin, ${ }^{1,2}$ Harsh V. Moolani, ${ }^{1}$ Abdoulaye Sene, ${ }^{1}$ Rohini Sidhu, ${ }^{3,4}$ Pamela Kell,, ${ }^{3,4}$ \\ Joseph B. Lin, ${ }^{1}$ Zhenyu Dong, ${ }^{1}$ Norimitsu Ban, ${ }^{1}$ Daniel S. Ory, ${ }^{3,4}$ and Rajendra S. Apte ${ }^{1,3,4,5}$ \\ 'Department of Ophthalmology and Visual Sciences, ${ }^{2}$ Neuroscience Graduate Program, Division of Biology and Biomedical \\ Sciences, ${ }^{3}$ Diabetic Cardiovascular Disease Center, ${ }^{4}$ Department of Medicine, and ${ }^{5}$ Department of Developmental Biology, \\ Washington University School of Medicine, St. Louis, Missouri, USA.
}

\begin{abstract}
Macrophage aging is pathogenic in diseases of the elderly, including age-related macular degeneration (AMD), a leading cause of blindness in older adults. However, the role of microRNAs, which modulate immune processes, in regulating macrophage dysfunction and thereby promoting age-associated diseases is underexplored. Here, we report that microRNA-150 (miR-150) coordinates transcriptomic changes in aged murine macrophages, especially those associated with aberrant lipid trafficking and metabolism in AMD pathogenesis. Molecular profiling confirmed that aged murine macrophages exhibit dysregulated ceramide and phospholipid profiles compared with young macrophages. Of translational relevance, upregulation of miR-150 in human peripheral blood mononuclear cells was also significantly associated with increased odds of AMD, even after controlling for age. Mechanistically, miR-150 directly targets stearoyl-CoA desaturase-2, which coordinates macrophage-mediated inflammation and pathologic angiogenesis, as seen in AMD, in a VEGF-independent manner. Together, our results implicate miR-150 as pathogenic in AMD and provide potentially novel molecular insights into diseases of aging.
\end{abstract}

License: This work is licensed under the Creative Commons Attribution 4.0 International License. To view a copy of this license, visit http:// creativecommons.org/licenses/ by/4.0/.

Conflict of interest: The authors have declared that no conflict of interest exists.

Submitted: January 25, 2018

Accepted: March 6, 2018

Published: April 5, 2018

\section{Reference information:}

JCI Insight. 2018;3(7):e120157. https://doi.org/10.1172/jici. insight.120157.

\section{Introduction}

Macrophages are critical effector cells of the innate immune system. Multiple groups, including our own, have reported that macrophages from aged mice demonstrate a functional drift compared with those isolated from young mice. For example, aged macrophages exhibit epigenomic changes, leading to reduced autophagic capacity (1), and are defective in their ability to fight viral infections due to reduced phagocytic activity (2). Moreover, aged macrophages are skewed toward a proangiogenic gene and cytokine expression profile, which leads to dysregulated inflammation and the inability to inhibit pathological angiogenesis (3). Aged macrophages also exhibit impaired cholesterol efflux due to decreased Abcal expression, leading to intracellular cholesterol accumulation and pathologic vascular proliferation (4). Age-associated macrophage dysfunction has been proposed to contribute to the pathogenesis of numerous diseases of aging, including age-related macular degeneration (AMD) and atherosclerosis (5). In addition, age-associated changes in microglia, the major resident immune cell in the retina with similar phagocytic functions, may also promote AMD (6).

AMD is a leading cause of blindness in industrialized nations (7) and displays a complex disease course characterized, initially, by accumulation of cholesterol-rich deposits known as drusen underneath the retina $(5,8)$. Though drusen themselves do not typically cause vision loss, they are risk factors for progression to one of 2 forms of advanced AMD: advanced neovascular (wet) AMD, characterized by pathologic subretinal angiogenesis, or advanced dry AMD, characterized by geographic atrophy secondary to loss of retinal neurons and underlying cells. Both forms of advanced AMD can cause debilitating blindness, though wet AMD causes a significant portion of the vision loss associated with AMD (9). While anti-VEGF therapies have revolutionized treatment options for wet AMD, an important subset of patients is un- or underresponsive to this therapy (8). Of interest, genome-wide association studies show that polymorphisms in lipid-related genes, including hepatic lipase (LIPC), ATP-binding cassette transporter member 1 (ABCA1), and cholesterol ester transfer protein (CETP), are associated with advanced AMD (10), supporting the idea that impaired cholesterol homeostasis contributes to AMD pathogenesis. 
Impaired cholesterol homeostasis also contributes to the pathogenesis of atherosclerosis. Atherosclerotic plaque formation begins when circulating monocytes adhere to the vascular endothelium, migrate to the sub-endothelial space, and activate into macrophages that take up lipids and become foam cells (11, 12). Past studies have demonstrated that the activation/polarization state of macrophages is important for predicting plaque phenotype and stability $(13,14)$. For example, in patients with hypercholesterolemia, macrophages polarize to a more proinflammatory state, which could predispose to plaque formation (15). Moreover, macrophage cholesterol efflux capacity in human patients is a clinically relevant predictor of atherosclerotic coronary artery disease (16), suggesting that perturbations in cholesterol homeostasis promote disease. Remarkably, atherosclerotic plaques and drusen have similar lipid compositions (17-20), unifying the pathogenic pathways underlying these diseases. Based on these similarities, some have proposed that it may be possible to repurpose statins, lipid-lowering drugs used to treat atherosclerosis, for treating $\operatorname{AMD}(21,22)$, although not all studies have yielded these same conclusions (23).

Despite these advances in our understanding of the phenotype of aged macrophages and how such changes contribute to age-associated diseases, the molecular mechanisms by which macrophages drift toward the disease-promoting phenotype remain elusive. Given the immense spectrum of these changes in aged macrophages, we hypothesized that microRNAs (miRs) may regulate the transcriptome of macrophages and, thereby, the transition of macrophages to a disease-promoting phenotype. The ability of miRs to target multiple genes makes them strong candidates as molecular regulators that skew macrophages toward a disease-promoting phenotype. Previous studies have examined the miR signatures of AMD by profiling eye fluids, such as human vitreous humor and plasma, providing phenotypic characterization, but they have failed to provide mechanistic insights into the underlying disease etiology $(24,25)$. Therefore, further elucidation of the target genes of these miRs, the affected cell types, and the molecular pathways involved is necessary for a more complete understanding of disease pathogenesis.

In this study, we sought to identify one or more miRs that regulate the disease-promoting programmatic changes in macrophages that are associated with AMD. Our results demonstrate that miR-150 is highly upregulated both in disease-promoting murine macrophages and in human peripheral blood mononuclear cells (PBMCs) from AMD patients. Moreover, we show that miR-150 regulates macrophage-mediated inflammation and pathologic angiogenesis independently from VEGF by targeting stearoyl-CoA desaturase-2 (Scd2), suggesting that it regulates the transition of macrophages from a healthy profile to the AMD-promoting phenotype. Ultimately, these findings provide insight into the mechanisms underlying the pathological programmatic changes in aged macrophages and may lead to the identification of novel therapeutic targets and candidate biomarkers.

\section{Results}

Aged macrophages have distinct cholesterol-responsive miR networks. Since miRs can regulate numerous target genes, we hypothesized that miRs may globally regulate the macrophage's response to exogenous cholesterol. We first sought to identify cholesterol-responsive miRs by performing a microarray, comparing untreated macrophages versus macrophages treated with acetylated LDL (acLDL) or oxidized LDL (oxLDL). We previously showed that aged and young macrophages handle cholesterol differently and that aged macrophages demonstrate altered capacity to metabolize cholesterol (4). As such, we profiled aged and young macrophages separately to accurately capture the response of these cells to exogenous cholesterol. In young macrophages, 10 miRs were similarly downregulated in response to both acLDL and oxLDL (Figure 1A). In contrast, in aged macrophages, 5 miRs were similarly dysregulated in response to both acLDL and oxLDL: 3 downregulated and 2 upregulated (Figure 1B). These findings suggest that, in macrophages, miRs are altered after exposure to cholesterol and may indeed orchestrate the macrophage's response to cholesterol. Furthermore, these findings suggest that the distinct miR responses in aged versus young macrophages may underlie their distinct responses to exogenous cholesterol.

In addition to these cholesterol-responsive miRs, we hypothesized that there may be a separate network of miRs that regulate the broad spectrum of differences between disease-promoting, aged macrophages and young macrophages. Such a miR must not only demonstrate altered expression with aging, but must also have consistent dysregulation regardless of treatment with cholesterol. Five miRs were upregulated in aged macrophages and maintained the same pattern of dysregulation in aged versus young macrophages after treatment with acLDL or oxLDL (Figure 1C). miR-150 was the strongest candidate for further study, given that it had the highest fold change in aged versus young macrophages and since the others did not validate after further characterization. 


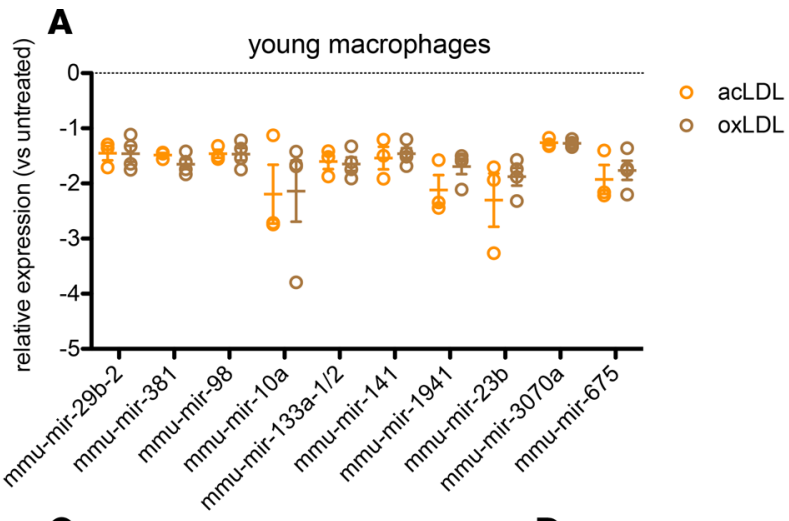

\section{C}

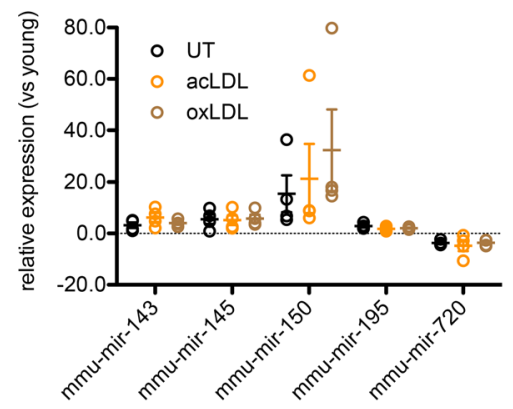

D

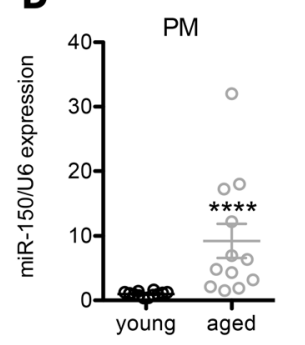

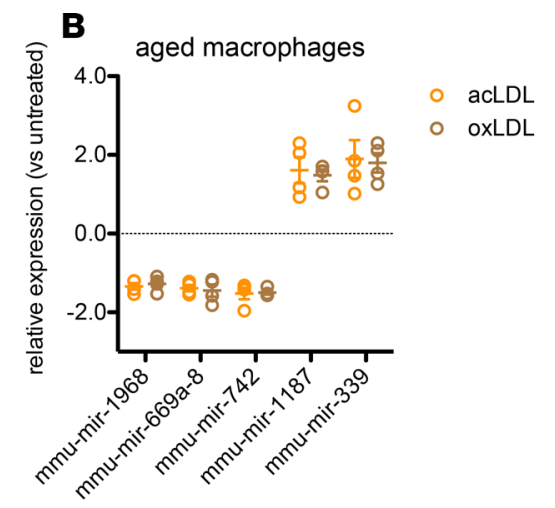

E

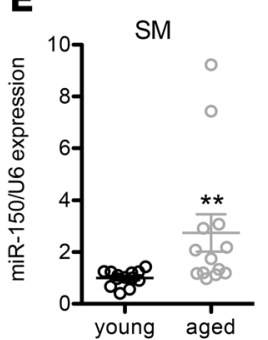

$\mathbf{F}$

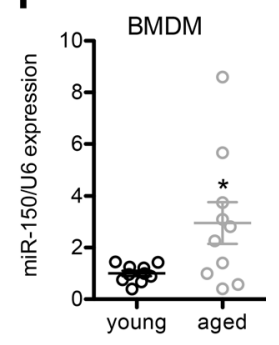

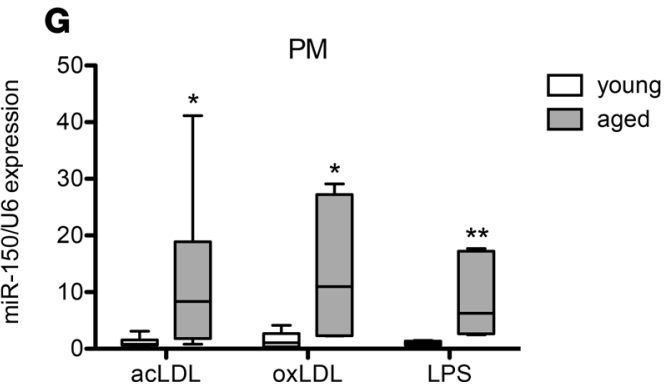

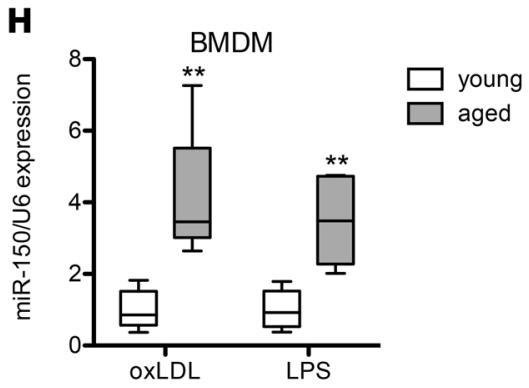

Figure 1. microRNA-150 is upregulated in aged macrophages of diverse origins. (A) In young macrophages, 10 microRNAs were downregulated similarly in response to acetylated low-density lipoprotein (acLDL) and oxidized low-density lipoprotein (oxLDL). (B) In aged macrophages, 5 microRNAs were dysregulated similarly in response to acLDL and oxLDL. (C) In untreated (UT), acLDL-treated, and oxLDL-treated macrophages, 5 microRNAs were dysregulated similarly in aged and young macrophages under the same treatment conditions. microRNA-150 was upregulated in aged peritoneal macrophages (PM) (D; $n=12$ / group; 2-tailed Mann-Whitney $U$ test), aged splenic macrophages (SM) (E; $n=13$ /group; 2-tailed Mann-Whitney $U$ test), and aged BM-derived macrophages (BMDM) (F; $n=10$ /group; 2-tailed, unpaired Welch's $t$ test). (C) Upregulation of microRNA-150 in aged PMs was not affected by treatment with acLDL ( $n$ $=7 /$ group; 2-tailed Mann-Whitney $U$ test), oxLDL ( $n=7 /$ group; 2-tailed, unpaired Welch's $t$ test), or LPS ( $n=5 /$ group; 2-tailed Mann-Whitney $U$ test). (H) Upregulation of microRNA-150 in aged BMDMs was not affected by treatment with oxLDL ( $n=5$ /group; 2-tailed Mann-Whitney $U$ test) or LPS ( $n=5 / g r o u p$; 2-tailed Mann-Whitney $U$ test). Open circles depict individual data points; graphs depict mean \pm SEM $(\mathbf{A}-\mathbf{F})\left({ }^{*} P<0.05 ;{ }^{* *} P<0.01 ;{ }^{* * * *} P<0.0001\right)$.

miR-150 is upregulated in aged macrophages of diverse origins. To validate the microarray data, we performed quantitative PCR (qPCR) with independent samples. Indeed, aged peritoneal macrophages (PMs) had 9-fold increased miR-150 expression at baseline compared with young PMs (Figure 1D). To determine whether this phenomenon was lineage specific, we also measured miR-150 expression in splenic macrophages (SMs) and BM-derived macrophages (BMDMs). Similar to PMs, we found that aged SMs (Figure 1E) and BMDMs (Figure 1F) exhibited 3-fold higher expression of miR-150 at baseline compared with young SMs and BMDMs. Next, we sought to confirm that miR-150 upregulation in aged macrophages is unaffected by exposure to exogenous cholesterol or LPS. Indeed, aged PMs treated with acLDL, oxLDL, or LPS exhibited higher miR-150 expression than similarly treated young PMs (Figure 1G). Likewise, oxLDL- and LPS-treated aged BMDMs also had higher miR-150 expression compared with similarly treated young BMDMs (Figure 1H). Cumulatively, these findings provide strong evidence that miR-150 upregulation may indeed skew macrophages toward the disease-promoting, aged phenotype. 
A

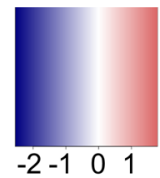

$\log _{2}$ fold change

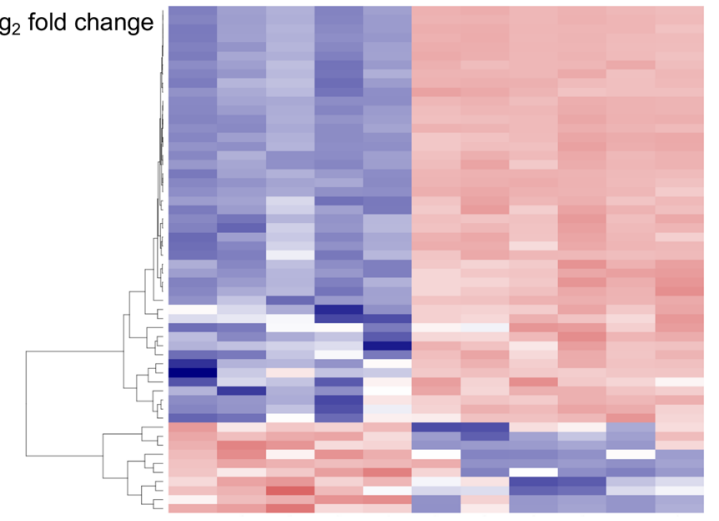

negative control miR-150

C

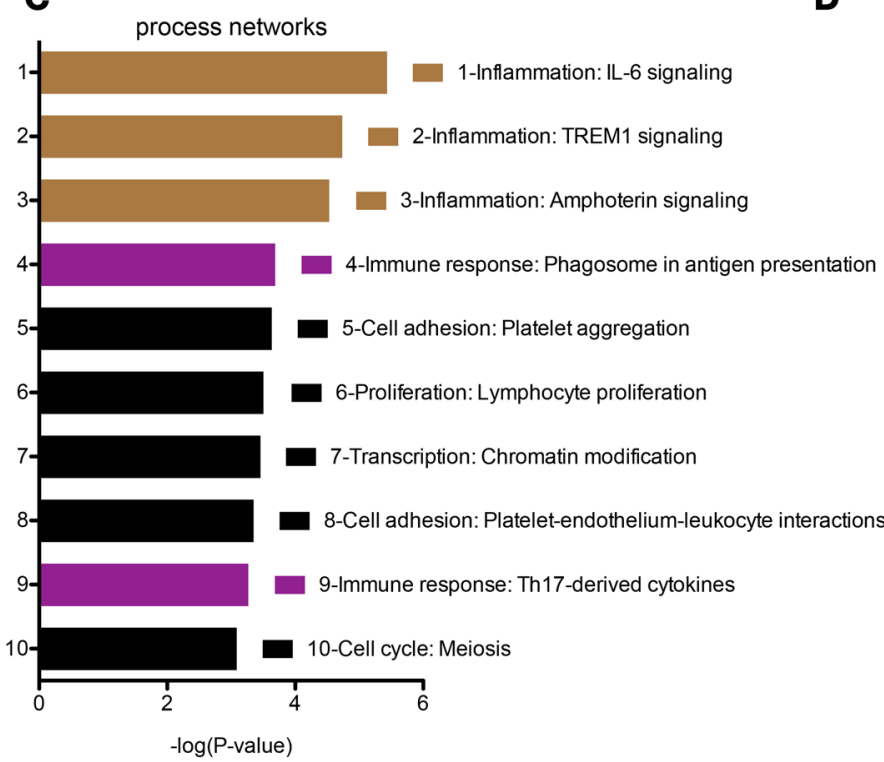

B

D
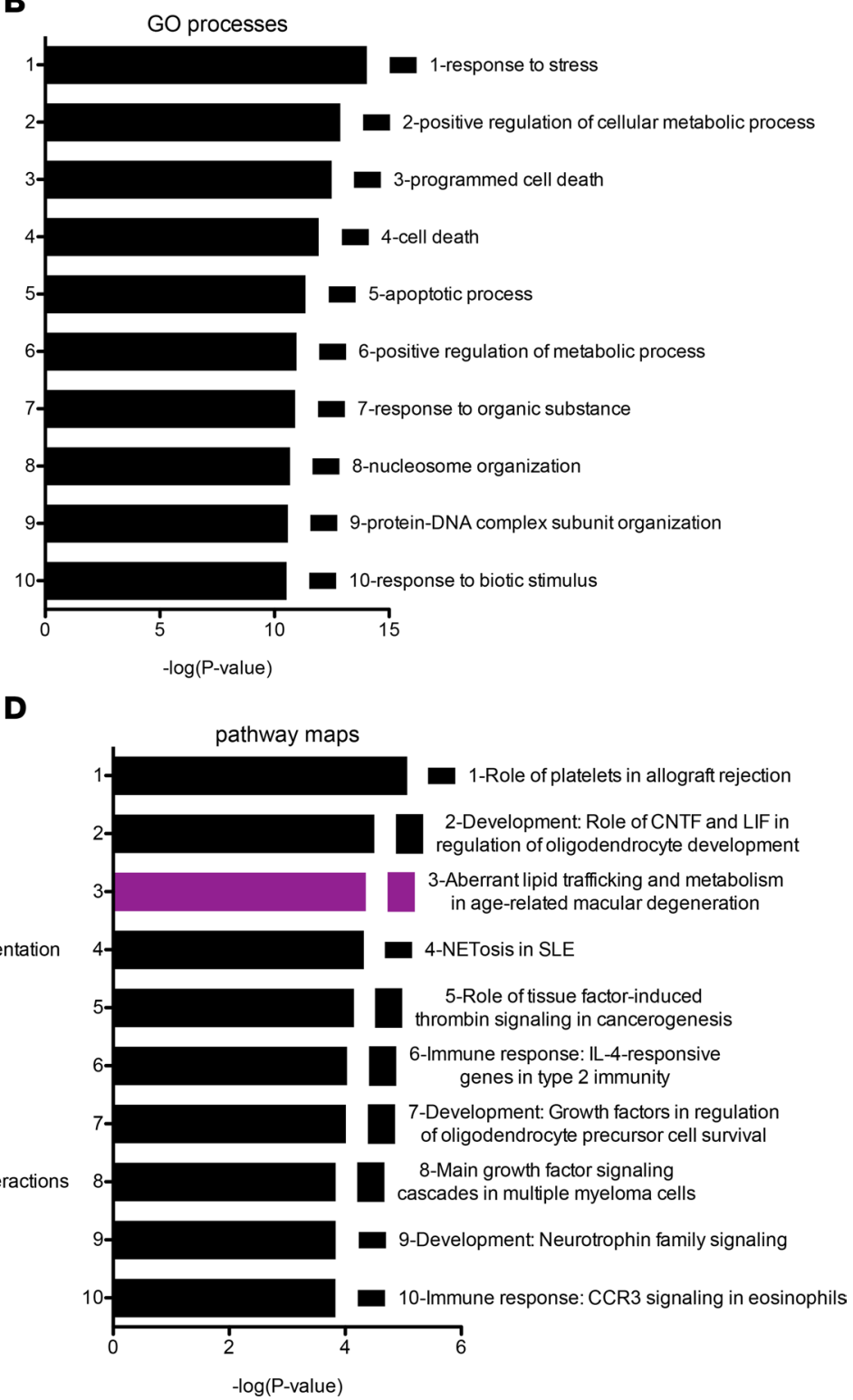

Figure 2. microRNA-150 (miR-150) regulates inflammation and lipid metabolism in macrophages. (A) RNA-sequencing followed by hierarchical clustering revealed clear transcriptomic differences between macrophages transfected with miR-150 mimic versus those transfected with a nontargeting negative control. Pathway analysis of the dysregulated genes in miR-150-overexpressing macrophages that are also dysregulated in aged macrophages (Jonathan B. Lin, unpublished observations) suggested perturbations in numerous gene ontology (G0) processes (B), process networks (C), and pathway maps (D). The altered transcriptomic profile of miR-150-overexpressing macrophages suggested dysregulation of numerous inflammation and immune response process networks ( $\mathbf{C}$; brown and purple, respectively) and aberrant lipid trafficking and metabolism in age-related macular degeneration (D; purple).

miR-150 overexpression reprograms the macrophage transcriptome. We next sought to determine the mechanism by which miR-150 skews macrophages toward the aged phenotype. To elucidate the cellular processes and pathways regulated by miR-150, we performed RNA sequencing (RNA-Seq) to identify the transcriptomic networks dysregulated under conditions of miR-150 overexpression, comparing young macrophages transfected with miR-150-overexpressing mimic ( $\mathrm{miR}-150^{\mathrm{OE}}$ ) to young macrophages transfected with a nontargeting negative control (NC) mimic. From RNA-Seq, we obtained an average of 35,607,314 reads per sample $(n=11)$, and of these, an average of $32,535,035$ reads $(91.4 \%)$ mapped to the mouse genome (Mus_musculus reference build Ensembl_R76). We performed hierarchical clustering, revealing clear differences between the transcriptomes of $\mathrm{miR}-150^{\mathrm{OE}}$ and NC-transfected PMs (Figure 2A). 
JClinsIGHT

RESEARCH ARTICLE
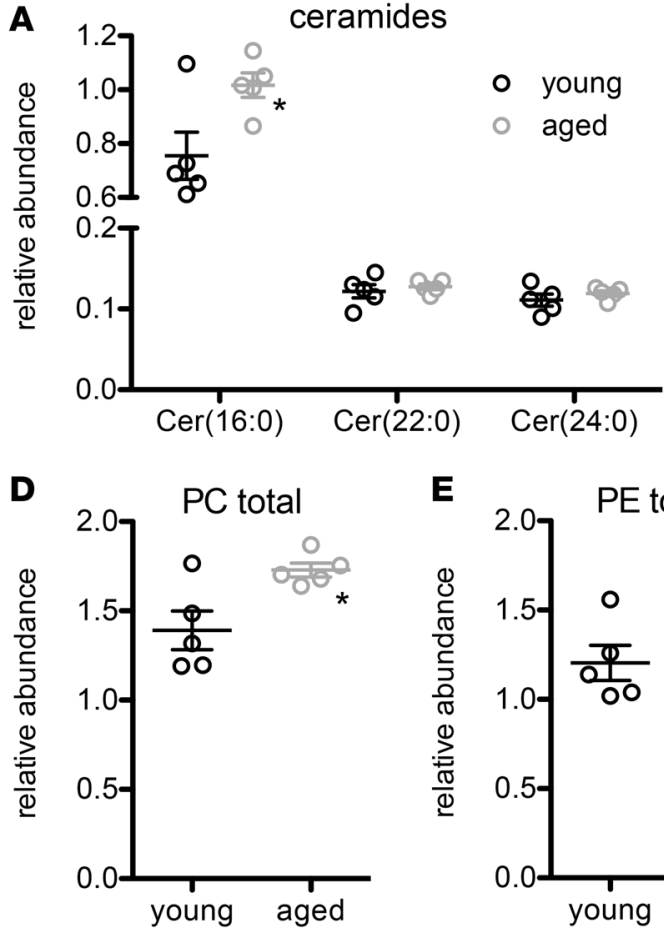

H

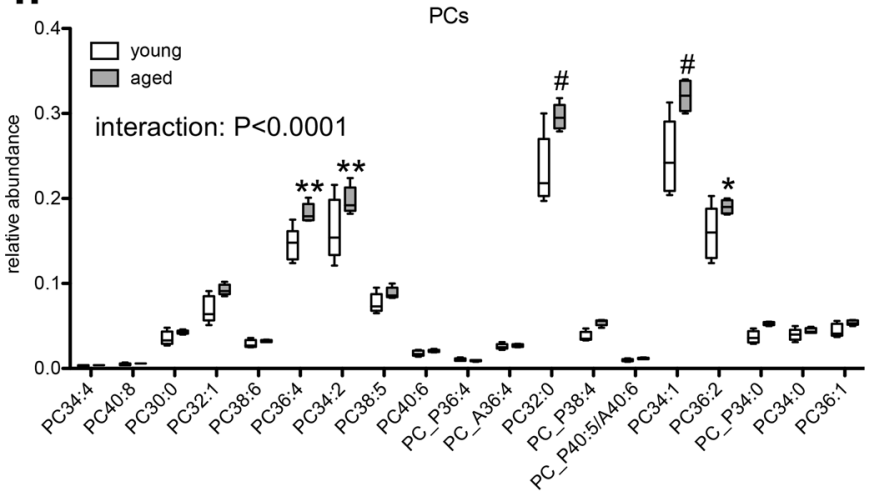

$\mathbf{J}$

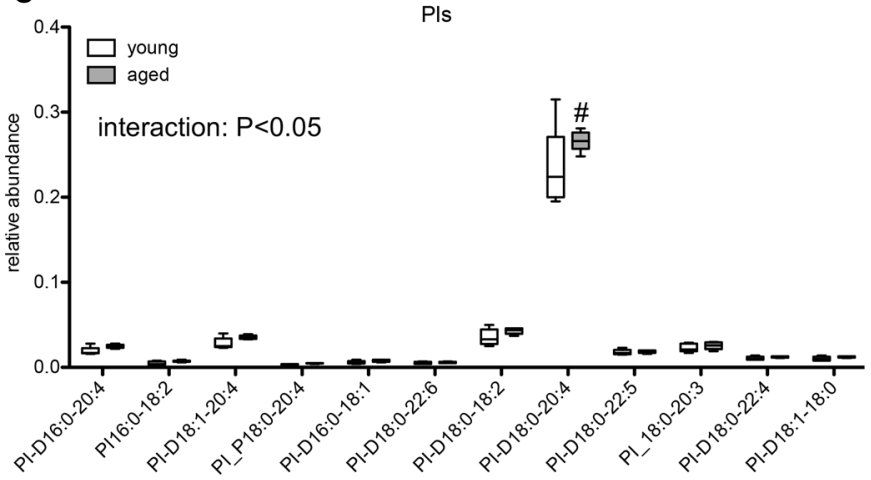

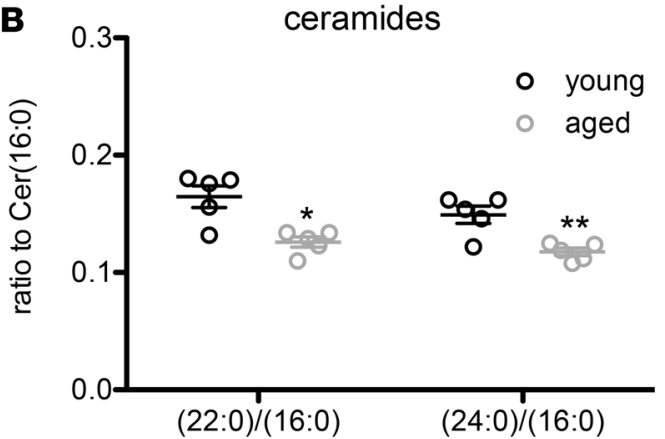

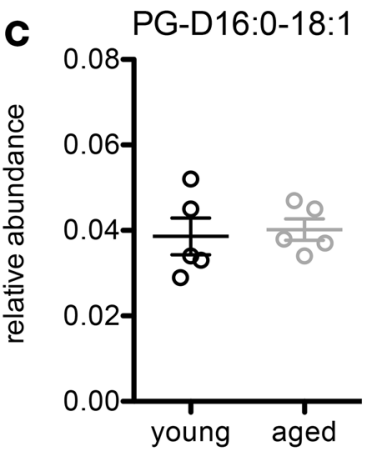

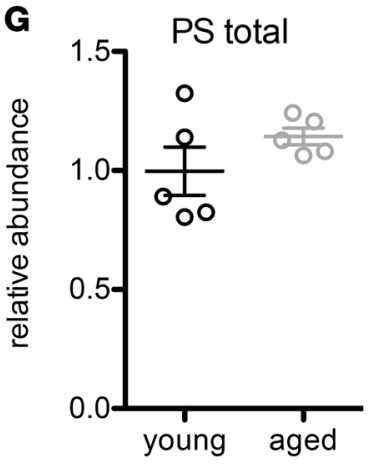

PEs

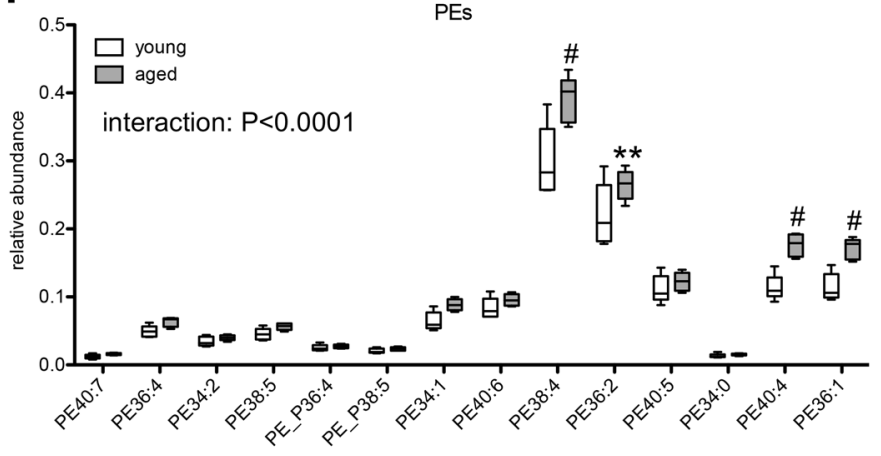

$\mathbf{K}$

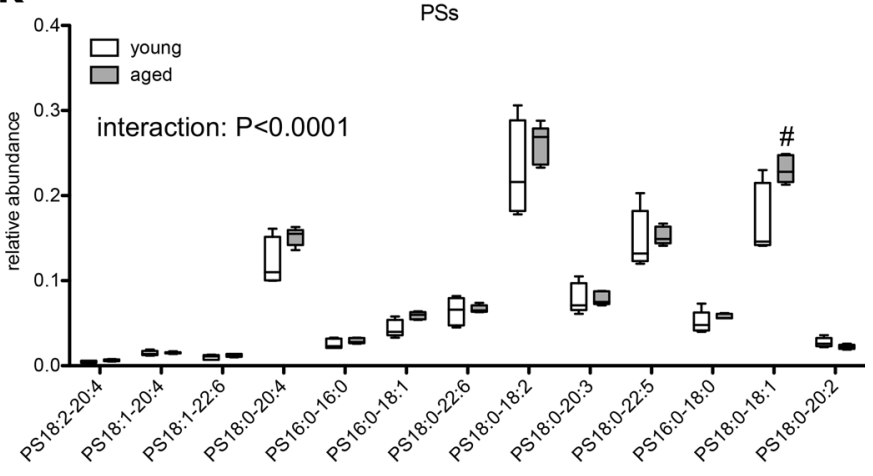

Figure 3. Aged macrophages have altered ceramide and phospholipid profiles. (A) Aged macrophages contained significantly more long-chain C16:0 than young macrophages but similar levels of very long-chain C22:0 and C24:0 ( $n=5$ /group; 2-tailed, unpaired Welch's $t$ test), resulting in decreased C22:0/ C16:0 and C24:0/C16:0 ratios (B; $n=5 /$ group; 2-tailed, unpaired Welch's $t$ test). Young and aged macrophages had similar phosphatidylglycerol-D16:0-18:1 (PG-D16:0-18:1) content ( $\mathbf{C} ; n=5$ /group; 2-tailed, unpaired student's $t$ test). Aged macrophages had higher total phosphatidylcholine (PC) (D; $n=5 / \mathrm{group}$; 2-tailed, unpaired student's $t$ test) and higher total phosphatidylethanolamine (PE) (E; $n=5$ /group; 2-tailed, unpaired student's $t$ test), but they had similar total phosphatidylinositol (PI) (F; $n=5$ /group; 2-tailed, unpaired Welsh's $t$ test) and similar total phosphatidylserine (PS) (G; $n=5 /$ group; 2-tailed, unpaired Welsh's $t$ test). Analysis of individual species revealed an interaction between age and species identity with increased levels of certain species but not others within each phospholipid class (H-K; $n=5$ /group; 2-way, repeated-measures ANOVA with Bonferroni post-hoc test). Open circles depict individual data points; graphs depict mean $\pm \operatorname{SEM}(\mathbf{A}-\mathbf{C})\left({ }^{*} P<0.05 ;{ }^{* *} P<0.01 ;{ }^{*} P<0.0001\right)$.

insight.jci.org https://doi.org/10.1172/jci.insight.120157

5 
Table 1. Demographic and clinical characteristics of human subjects

\begin{tabular}{|c|c|c|c|}
\hline Variable & Control & AMD & $P$ value \\
\hline Age, mean (SD) & $64.57(10.27)$ & $75.48(12.77)$ & $<0.001^{A}$ \\
\hline \multicolumn{4}{|l|}{ Sex, $n$} \\
\hline Male & 36 & 20 & $0.282^{B}$ \\
\hline Female & 27 & 23 & \\
\hline \multicolumn{4}{|l|}{ AMD status, $n$} \\
\hline No AMD & 63 & 0 & $N / A$ \\
\hline Early AMD & 0 & 20 & \\
\hline Advanced neovascular (wet) AMD & 0 & 23 & \\
\hline
\end{tabular}

To determine the subset of these miR-150-regulated genes that are also dysregulated in aged macrophages, we overlaid the RNA-Seq results onto the results of a previous microarray that we performed comparing aged versus young macrophages (Jonathan B. Lin, unpublished observations). The intersection between these gene lists identifies genes dysregulated in aged murine macrophages that may be regulated by miR-150 upregulation. We identified 160 commonly dysregulated genes with a |fold change| $>1.2$ both in $\mathrm{miR}-150^{\mathrm{OE}}$ versus NC-transfected macrophages and in aged versus young macrophages. To determine whether these genes suggested abnormalities in specific pathways, which may provide insight into the mechanism by which miR-150 upregulation promotes age-associated disease, we performed pathway analysis with MetaCore for enrichment by gene ontology (GO) processes, process networks, and pathway maps (Figure 2, B-D). Numerous diverse GO processes were enriched for, indicating that miR-150 regulates broad cellular pathways in macrophages (Figure 2B). Of interest, inflammation and immune response process networks were 5 of the 9 most enriched process networks (Figure 2C). These findings confirm that there is immune dysregulation in aged macrophages associated with miR-150 upregulation. Remarkably, aberrant lipid trafficking and metabolism in AMD was the third most enriched pathway map (Figure 2D), validating our hypothesis that miR-150 regulates the transition of macrophages to the AMD-promoting phenotype.

Disease-promoting macrophages have altered phospholipid and ceramide profiles. In AMD, the interaction between dysregulated macrophage cholesterol homeostasis and aging is implicated in the pathophysiology of disease. To better understand this relationship, we performed lipidomics to determine whether aged macrophages have altered lipid profiles as possible consequences of aberrant lipid trafficking and metabolism. We focused on components of the plasma membrane, including ceramides and phospholipids, since the composition and organization of the plasma membrane have been shown to regulate macrophage function (26). Aged macrophages contained significantly more long-chain ceramide (C16:0) than young macrophages but similar levels of very long-chain C22:0 and C24:0 (Figure 3A). Consistent with increased C16:0, we observed a significant decrease in both the $C 22: 0 / C 16: 0$ and $C 24: 0 / C 16: 0$ ratios in aged versus young macrophages (Figure 3B). These findings indicate that aged macrophages exhibit remodeling of their ceramide composition from very long-chain to long-chain species.

In the phosphatidylglycerol (PG) class, PG-D16:0-18:1 was the only detectable species, and there was no significant difference in content between aged and young macrophages (Figure 3C). However, numerous individual species were detected for each of the other phospholipid classes. Overall, aged macrophages contained more total phosphatidylcholine (PC; Figure 3D) and more total phosphatidylethanolamine (PE; Figure 3E) than young macrophages but similar total phosphatidylinositol (PI; Figure 3F) and similar total phosphatidylserine (PS; Figure 3G). Further analysis of the individual species within each phospholipid class by 2-way, repeated-measures ANOVA revealed a significant interaction between the main effects of species identity and age for all 4 phospholipid classes (Figure 3, H-K), indicating that the increased total PC and total PE in aged macrophages were driven by increases in specific species within these phospholipid classes. Post hoc testing showed that the significantly increased species were generally phospholipids containing fatty acids with multiple double bonds, suggesting a shift toward unsaturated fatty acids (Figure 3, H-K). Based on these findings, we propose that aberrant lipid trafficking and metabolism in aged macrophages leads to this disruption in ceramide and phospholipid profiles, which may contribute to macrophage dysfunction in age-associated diseases. 

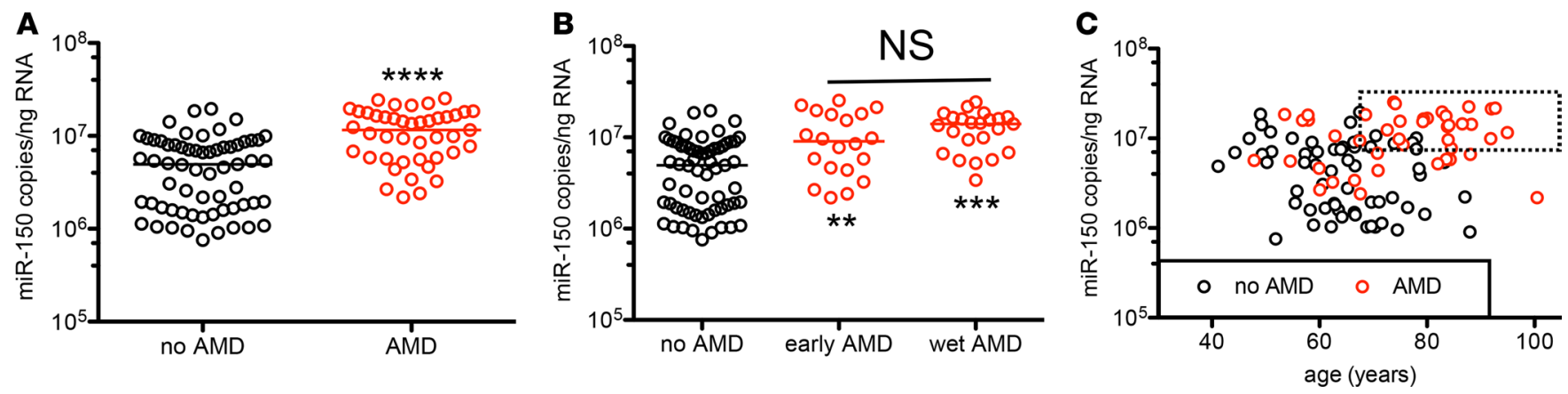

Figure 4. Upregulation of microRNA-150 in human peripheral blood mononuclear cells (PBMCs) is associated with age-related macular degeneration (AMD). (A) AMD patients $(n=43)$ had higher PBMC microRNA-150 copy numbers compared with controls ( $n=63 ; 2$-tailed Mann-Whitney $U$ test). (B) Both early AMD $(n=20)$ and wet AMD patients $(n=23)$ had higher PBMC microRNA-150 copy numbers compared with controls $(n=63)$, but there was no significant difference between early and wet AMD patients (Kruskal-Wallis test with Dunn's multiple comparison post-hoc test). (C) There was no correlation between PBMC microRNA-150 copy number and age in AMD patients or controls. Patients in the highest tertile of microRNA-150 copy number and above median age, as indicated by the dashed rectangle, had the highest prevalence of AMD (87.5\%). Open circles depict individual human subjects (A-C); horizontal lines depict medians (A and $\mathbf{B})\left({ }^{* *} P<0.01 ;{ }^{* *} P<0.001 ;{ }^{* * *} P<0.0001\right)$.

miR-150 upregulation is associated with AMD in humans. To assess the translational relevance of these findings, we recruited human patients with early AMD or advanced neovascular (wet) AMD and non-AMD controls in a case-control study design. Demographic and clinical information of the participants are shown in Table 1. From these patients, we collected PBMCs, which contain mostly monocytes, and quantified miR-150 copy number in these samples. On average, AMD patients had significantly higher PBMC miR-150 levels compared with control subjects (Figure 4A). When we subdivided AMD patients into early AMD and wet AMD groups, we observed higher PBMC miR-150 levels in early AMD patients compared with controls and in wet AMD patients compared with controls (Figure 4B). There was, however, no significant difference in PBMC miR-150 levels between early and wet AMD patients (Figure 4B). These findings suggest that increased PBMC miR-150 levels are associated with developing AMD but not with disease progression. We next sought to determine whether age affected PBMC miR-150 levels, since AMD patients tended to be older than nonAMD controls (Table 1). We found that there was no significant correlation between age and PBMC miR-150 levels in AMD patients $\left(r_{s}=0.1596, P=0.3066\right)$ or in control subjects $\left(r_{s}=-0.2002, P=0.1157\right)$ (Figure 4C), suggesting that there is no significant association between PBMC miR-150 levels and age.

To model the relationship between PBMC miR-150 levels and AMD status and to rigorously control for a possible - albeit unlikely - effect of age, we generated a binary logistic regression model with the predictor variables of age, sex, and $\log _{10}$-transformed PBMC miR-150 levels and the outcome of AMD. The overall model was statistically significant ( $\mathrm{LR} \chi^{2}=47.4$, degrees of freedom $\left.[\mathrm{df}]=3, P<0.001\right)$ and had good fit $\left(\chi^{2}=9.4\right.$, df $=8, P=0.311)$. As expected, age was a significant predictor of $\operatorname{AMD}(P<0.001)$ with a $\beta$ coefficient of 0.082 , indicating that each additional year of age was associated with increased odds of having AMD (adjusted odds ratio $[\mathrm{aOR}]=1.086 ; 95 \% \mathrm{CI}, 1.04-1.13)$. Of significant interest, even after controlling for the effects of age and sex on miR-150 levels, $\log _{10}$-transformed PBMC miR-150 levels were highly associated with AMD $(P<0.001)$ with a $\beta$ coefficient of 3.367, indicating that each additional 10-fold increase in PBMC miR-150 levels was associated with 29.0-fold increased odds of having AMD (95\% CI, 5.9-141.5). To determine the efficacy of PBMC miR-150 levels as a potential marker of AMD, we generated a receiver operating characteristic (ROC) curve and found that the area under the ROC curve was 0.860 (95\% CI, 0.788-0.933), indicating good discrimination.

Furthermore, we performed conjunctive analysis to illustrate the relationship between PBMC miR-150 levels, age, and the outcome of AMD. We divided patients into tertiles by PBMC miR-150 levels (cutoffs of $4.5 \times 10^{6}$ and $9.5 \times 10^{6}$ copies/ng RNA) and by age (i.e., above versus below a median of 67.47 years), tabulating the AMD prevalence in each conjoined cell (Table 2). This analysis demonstrated a clear stepwise increase in prevalence of AMD going from the lowest to the highest tertile of PBMC miR-150 levels in participants both above median age (i.e., from $18.8 \%$ to $53.8 \%$ to $87.5 \%$ ) and below median age (i.e., from $15.8 \%$ to $17.4 \%$ to $45.5 \%$ ). These findings strongly support the notion that increased PBMC miR-150 levels are associated with AMD. This trend held true both above and below median age, making it highly unlikely that the differences in PBMC miR-150 levels in AMD patients versus non-AMD controls were solely due to the fact that the AMD patients were, on average, older. 
Table 2. Age-related macular degeneration as a function of age and peripheral blood mononuclear cell (PBMC) microRNA-150 (miR-150) levels

\begin{tabular}{|c|c|c|c|c|}
\hline & & \multicolumn{3}{|c|}{ Age } \\
\hline & & Below median & Above median & Total \\
\hline \multirow{4}{*}{ PBMC miR-150 } & Lowest tertile & $3 / 19(15.8 \%)$ & $3 / 16(18.8 \%)$ & 6/35 (17.1\%) \\
\hline & Middle tertile & $4 / 23(17.4 \%)$ & $7 / 13(53.8 \%)$ & $11 / 36$ (30.6\%) \\
\hline & Highest tertile & $5 / 11(45.5 \%)$ & $21 / 24$ (87.5\%) & $26 / 35(74.3 \%)$ \\
\hline & Total & $12 / 53(22.6 \%)$ & $31 / 53$ (58.5\%) & $43 / 106(40.6 \%)$ \\
\hline
\end{tabular}

miR-150 directly targets $S c d 2$ and promotes pathologic angiogenesis. To understand the mechanism by which miR-150 promotes macrophage dysfunction, we sought to identify direct miR-150 targets. Since miRs canonically downregulate their gene targets, we filtered for commonly downregulated genes (fold change $<-1.2$ ) both in miR-150 ${ }^{\mathrm{OE}}$ and NC-transfected macrophages and in aged versus young macrophages. This strategy identified 36 initial putative target genes (Supplemental Table 1; supplemental material available online with this article; https://doi.org/10.1172/jci.insight.120157DS1). We further narrowed this list by eliminating genes that either did not appear in any of 6 target identification databases or did not contain a seed sequence in their 3' UTR. We performed qPCR in aged versus young macrophages on the remaining 26 genes. Of these 26 genes, 8 showed significant downregulation in aged macrophages (Figure $5, \mathrm{~A}$ and B). Of interest, 2 of the 8 genes that were significantly downregulated in aged macrophages are known to play key roles in fatty acid biosynthesis: fatty acid synthase (Fasn) and $S c d 2$. We confirmed that both genes were indeed downregulated in miR- $150^{\mathrm{OE}}$ macrophages versus NC-transfected macrophages (Figure 5, C and D). To determine the functional effect of Fasn and $S c d 2$ deficiency, we performed siRNA knockdown of these gene targets in macrophages and assessed the inflammation status and angiogenic potential of these macrophages by PCR array. We confirmed siRNA knockdown of Fasn and Scd2 by qPCR (Figure 5, E and F). Fasn-deficient macrophages exhibited upregulation of only Ptgs 2 and Tnf(Figure 5G) and no upregulation of proangiogenic factors (Figure $5 \mathrm{H}$ ). On the other hand, $S c d 2$-deficient macrophages were abnormally activated, with upregulation of numerous proinflammatory markers (Figure 5I) and proangiogenic factors (Figure 5J). Of interest, Vegfa expression was not altered (Figure 5J), suggesting that Scd2-mediated regulation of macrophage function is independent from VEGF. These results suggest that $S c d 2$ may be an important direct target of miR-150, providing a mechanism by which miR-150 regulates macrophage function.

Next, we performed dual-reporter assays to confirm that miR-150 can directly regulate $S c d 2$ expression. The $S c d 2$ gene contains 2 canonical miR-150 seed sequence target sites in its 3' UTR: a 7mer-A1 (3' - UGGGAGA - 5') and an offset 6mer (3' - UUGGGA - 5') (Figure 6A). As expected, cotransfection of a plasmid with the $S c d 23^{\prime}$ UTR cloned downstream of a secreted Gaussia luciferase (GLuc) and miR-150 mimic led to decreased GLuc activity compared with cotransfection of the same plasmid with a nontargeting mimic (Figure 6B). Removing the 7mer-A1 target site (mutant 1) significantly reduced the extent to which miR150 cotransfection reduced GLuc activity (Figure 6C). In contrast, removing the offset 6mer target site (mutant 2) did not change the negative regulatory effect of miR-150 cotransfection on GLuc activity (Figure $6 \mathrm{C}$ ). These findings suggest that the 7 mer-A 1 site is the dominant target site to which miR-150 binds to regulate $S c d 2$ expression, while the offset 6 mer site plays a less important role.

To determine whether downregulation of $S c d 2$ may promote pathological angiogenesis, as seen in wet AMD, we measured the extent to which $S c d 2$-deficient macrophages inhibited choroidal neovascularization (CNV) in a well-established murine model of injury-induced angiogenesis in the eye. Although host macrophages play an important role in regulating CNV, we have previously demonstrated that intravitreal injection of functional macrophages can augment the antiangiogenic effect (27). Therefore, we performed laser injury to induce CNV and injected $S c d 2$-deficient or NC-transfected macrophages intravitreally by adoptive transfer immediately after injury. Adoptively transferred $S c d 2$-deficient macrophages were not able to inhibit CNV as effectively as NC-transfected macrophages (Figure 6, D and E). This phenomenon was independent of VEGF since VEGF mRNA expression and protein secretion was not increased in Scd2-deficient macrophages (Figure $5 \mathrm{H}$ and Figure $6 \mathrm{~F}$ ). To confirm these in vivo results, we also generated mice lacking $S c d 2$ in myelomonocytic cells, including macrophages, with the Cre-lox system $\left(S c d 2^{-\mathrm{m} /-\mathrm{m}}\right)$. In agreement 

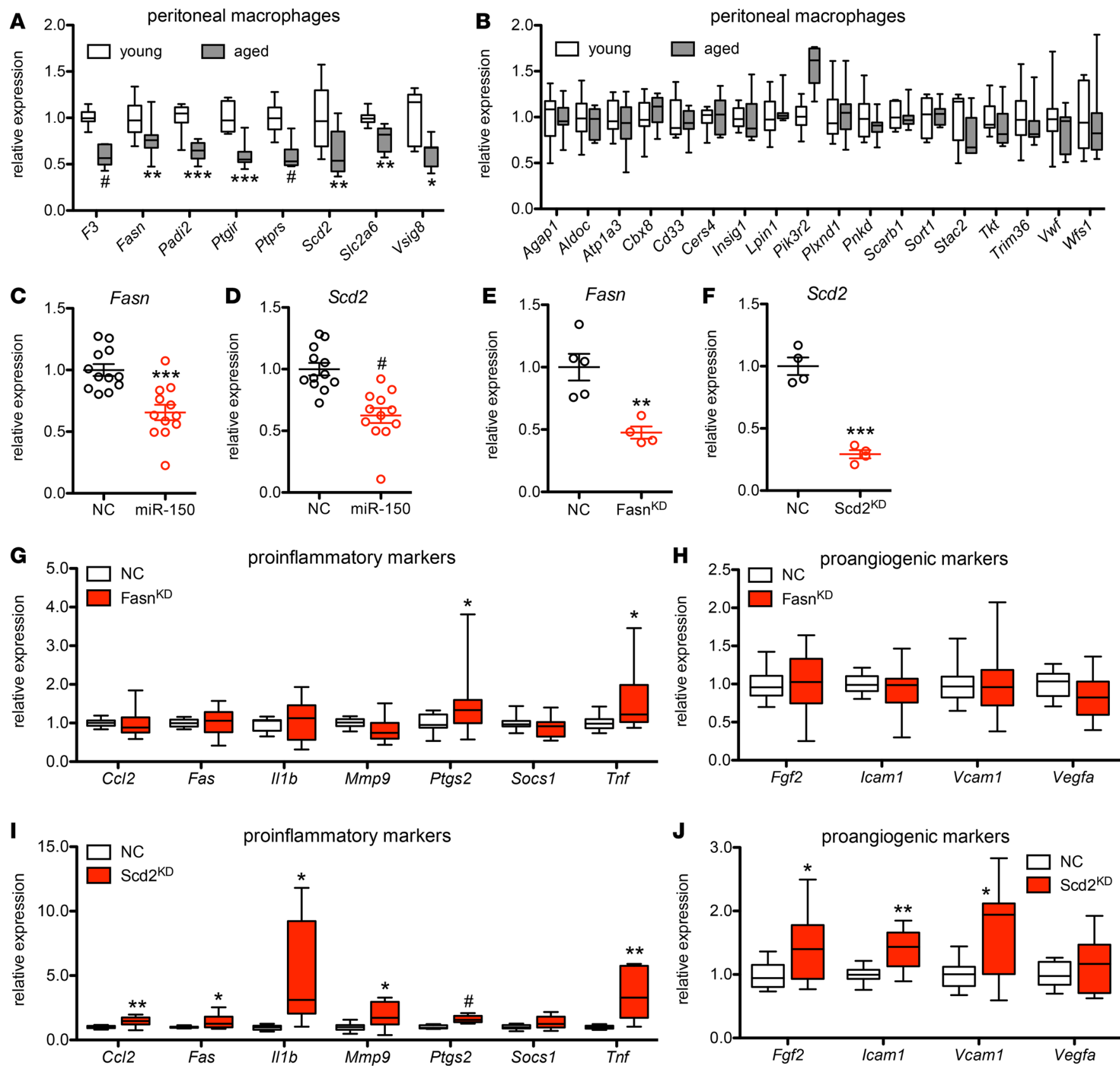

Figure 5. microRNA-150 modulates fatty acid synthase (Fasn) and stearoyl-CoA desaturase-2 (Scd2) expression. (A and B) Eight of the 26 putative microRNA-150 targets had decreased expression in aged macrophages ( $n=6-12 /$ group; 2-tailed, unpaired Welch's $t$ test). (C and D) microRNA-150 mimic-transfected macrophages had reduced expression of Fasn and Scd2 compared with nontargeting negative control-transfected (NC-transfected) macrophages ( $n=12$ /group; 2-tailed, unpaired student's $t$ test). (E and F) Macrophages transfected with Fasn-and Scd2-targeting small-interfering RNA (siRNA) had reduced expression of target genes ( $n=4-5 /$ group; 2-tailed, unpaired student's $t$ test; KD, knock down). (G and H) Fasn-deficient $\left(\mathrm{Fasn}^{\mathrm{KD}}\right)$ macrophages were somewhat abnormally activated but had normal expression of proangiogenic factors ( $n=14 / \mathrm{group}$; 2-tailed, unpaired Welch's $t$ test). (I and J) Scd2-deficient (Scd2 ${ }^{\mathrm{KD}}$ ) macrophages were abnormally activated and had increased expression of proangiogenic factors ( $n=10$ /group; 2-tailed, unpaired Welch's $t$ test). Open circles depict individual data points; graphs depict mean \pm SEM $(\mathbf{C}-\mathbf{F})\left({ }^{*} P<\right.$ $\left.0.05 ;{ }^{*} P<0.01 ;{ }^{* *} P<0.001 ;{ }^{*} P<0.0001\right)$.

with our adoptive transfer experiments, $S c d 2^{-\mathrm{m} / \mathrm{m}}$ mice had larger $\mathrm{CNV}$ complexes after laser injury compared with floxed controls ( $S c d 2^{\mathrm{f} / \mathrm{f}}$ ) (Figure 6, G and H). In contrast, mice lacking Fasn in myelomonocytic cells $\left(\right.$ Fasn $\left.^{-\mathrm{m} / \mathrm{m}}\right)$ and floxed controls $\left(\right.$ Fasn $\left.^{\mathrm{f} / \mathrm{f}}\right)$ had similar CNV complex sizes after laser injury (Figure 6, I and J). These findings confirm that $S c d 2$ plays an important role in regulating macrophage function in the context of pathological angiogenesis. 
A

\section{0 : 5'...gccgugggagagggg...3' \\ 3'-gugaccauguucccaacccucu-5' \\ miR-150-5p}

Scd2 3' UTR

Scd2 3' UTR

2,774: 5'...cguuuugggaa cuu...3'

|| |1।

3' -gugaccauguucccaacccucu-5'

miR-150-5p
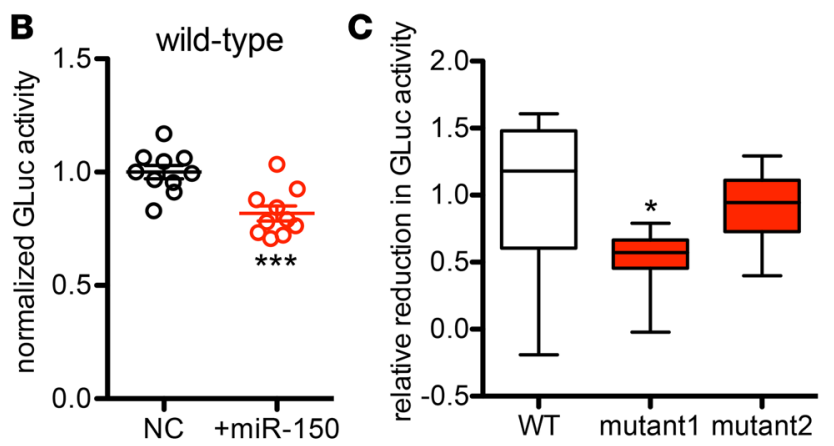

D
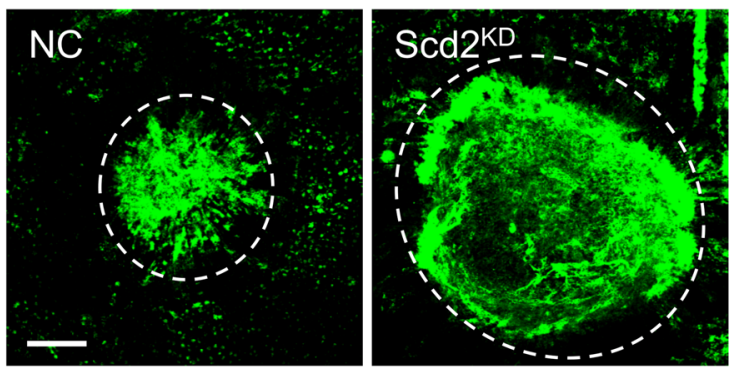

E
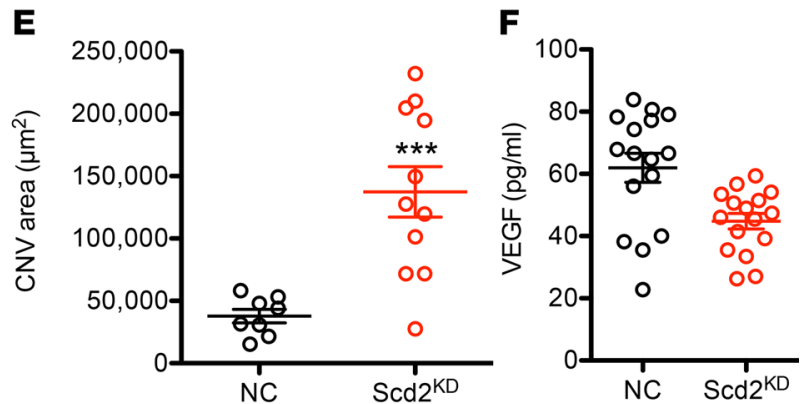

G
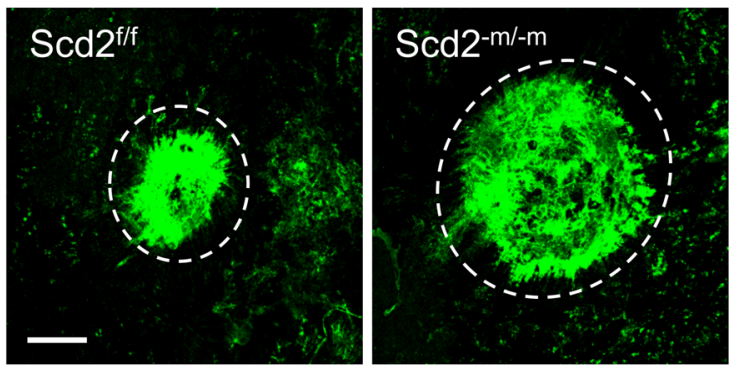

$\mathbf{H}$

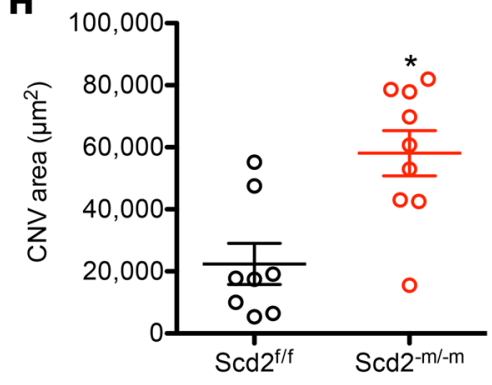

I
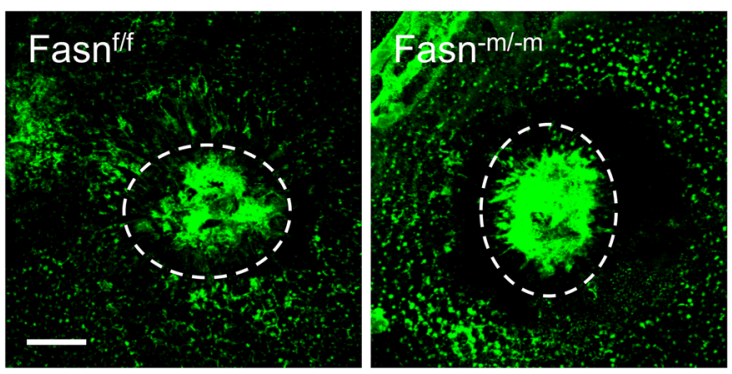

J

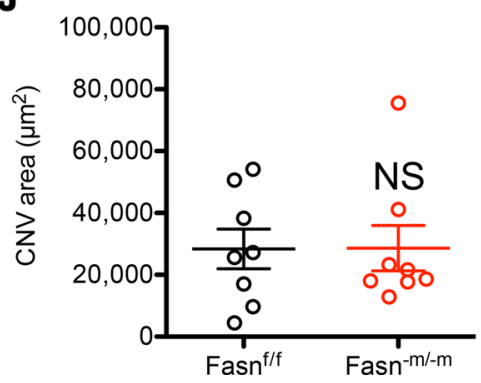

Figure 6. microRNA-150 directly targets Scd2 and thereby promotes pathological angiogenesis. (A) The 3' untranslated region (UTR) of Scd2 contains canonical 7mer-A1 and offset 6mer microRNA-150 binding sites. (B) Cotransfection of a dual-reporter plasmid with the Scd2 3' UTR inserted downstream of a secreted Gaussia luciferase (GLuc) reporter gene and microRNA-150 mimic led to reduced GLuc activity compared with cotransfection of the same plasmid with a negative control (NC) mimic ( $n=10 /$ group; 2-tailed, unpaired student's $t$ test). (C) Removing the $7 \mathrm{mer-A} 1$ target site (mutant 1 ) but not the offset 6 mer target site (mutant 2 ) reduced the extent to which microRNA-150 cotransfection inhibited GLuc activity ( $n=10$ / group; Kruskal-Wallis test with Dunn's multiple comparison post-hoc test). (D and E) Adoptively transferred Scd2-deficient (Scd2 ${ }^{\mathrm{KD}}$ ) macrophages were less able to inhibit laser injury-induced choroidal neovascularization (CNV) compared with NC-transfected macrophages (D, representative images from $n=8-11$ burns/group; 2-tailed, unpaired Welch's $t$ test). (F) In vitro VEGF secretion was not significantly increased in Scd2-deficient macrophages ( $n=16 /$ group; 2-tailed, unpaired Welch's $t$ test). (G and H) Scd2-m/-m mice exhibited larger CNV complexes after laser injury compared with $S c d 2^{f / f}$ mice (G, representative images from $n=8-9$ burns/group; 2-tailed Mann-Whitney $U$ test). (I and J) Fasn ${ }^{-m /-m}$ and Fasn ${ }^{f / f}$ mice had similarly sized CNV complexes after laser injury (I, representative images from $n=8$ burns/group; 2-tailed Mann-Whitney $U$ test). Scale bars: $100 \mu m$ (D, G, I). Open circles depict individual data points; graphs depict mean \pm SEM (B, E, F, H, J) $\left({ }^{*} P<0.05 ;{ }^{* *} P<0.001\right)$. 


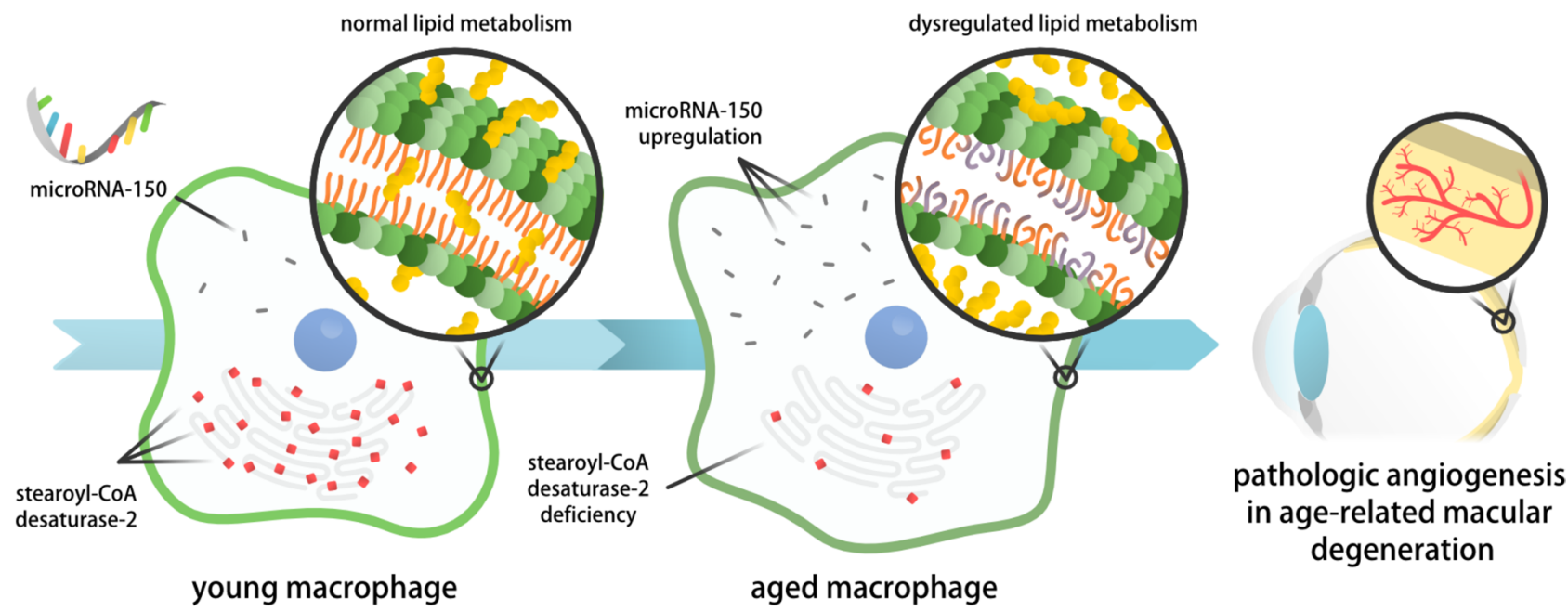

Figure 7. Upregulation of miR-150 in aged macrophages causes stearoyl-CoA desaturase-2 deficiency and dysregulated lipid metabolism, thereby promoting pathological angiogenesis, as seen in age-related macular degeneration.

Together, these findings indicate that $S c d 2$ downregulation secondary to miR-150 upregulation in aged macrophages promotes macrophage dysfunction and pathological angiogenesis, providing a mechanism by which miR-150 can direct macrophages toward an aged, disease-promoting, and proangiogenic phenotype (Figure 7). Our human data confirm the translational relevance of these findings in AMD pathogenesis.

\section{Discussion}

In this study, we report that miR-150 is upregulated in aged murine macrophages of diverse origins and directs aged macrophages toward a disease-promoting phenotype characterized by abnormal activation and promotion of pathologic angiogenesis. miR-150 was previously reported to play important roles in controlling B cell differentiation by targeting the transcription factor c-Myb (28). Moreover, miR-150 has been reported to regulate de novo lipogenesis by targeting Fasn and other lipid-related genes in mammary epithelium (29). Here, we uncover a potentially novel role for miR-150 in macrophages to regulate cholesterol metabolism and lipid trafficking genes involved in AMD based on our RNA-Seq results. In agreement, other miRs have been shown to regulate cholesterol homeostasis. For example, miR-33 (4, 30), miR-302a (31), and miR-19b (32) regulate reverse cholesterol transport by modulating Abca1 expression.

Consistent with their impaired cholesterol metabolism and lipid trafficking, we report that aged macrophages with miR-150 upregulation have associated alterations in ceramide and phospholipid profiles. These results build on our previous finding that aged macrophages have impaired cholesterol efflux (4). Specifically, aged macrophages have a reduced ratio of very long-chain to long-chain ceramides. Ceramides are important signaling molecules in macrophages that modulate cellular responses in many pathways, including inflammation (33). Different molecular ceramide species have been shown to be significantly associated with mortality in coronary artery disease patients independently of traditional risk factors (34), highlighting their importance in disease pathogenesis. Additionally, we demonstrate that aged macrophages have alterations in phospholipid composition in multiple classes. Of interest, both ceramides and phospholipids are important components of the plasma membrane, and the composition and organization of the plasma membrane have been shown to be important for modulating cholesterol-dependent signaling networks involved in inflammation (26).

We propose that broad disruptions in plasma membrane lipids secondary to miR-150 upregulation in aged macrophages modulate the inflammatory status of aged macrophages and thereby predispose them toward a disease-promoting, proangiogenic phenotype. Our lipidomic analysis shows that aged macrophages possess a complex profile of altered lipid composition with remodeling toward long-chain ceramides and a shift toward phospholipids containing unsaturated fatty acids. We provide further insight by demonstrating that 1 molecular mechanism by which miR-150 regulates lipid metabolism in macrophages is direct targeting of $S c d 2$. This gene catalyzes the rate-limiting step in the formation of monounsaturated 
fatty acids and has been shown to be important in lipid synthesis during early skin and liver development (35). Our data establish that $S c d 2$ is also evidently important in macrophage function, as $S c d 2$-deficient macrophages exhibit abnormal activation and promote pathological angiogenesis. Although Scd2 deficiency alone might be expected to cause accumulation of phospholipids containing saturated fatty acids in aged macrophages, we did not observe this pattern in our lipidomic analysis, suggesting that $S c d 2$ deficiency does not drive alterations in lipid composition alone. We propose instead that the combination of Scd2 deficiency and changes in other lipid-related genes causes the altered lipid profile of aged macrophages.

Of translational relevance, our study provides strong evidence that miR-150 plays a role in AMD pathogenesis. Specifically, our data indicate not only that AMD patients have higher PBMC miR-150 levels than control participants, but also that increased PBMC miR-150 is significantly associated with increased odds of AMD in a sex- and age-adjusted binary logistic regression model. Although miR-150 levels were associated with disease in human PBMCs, there was no clear relationship between miR-150 levels and age, unlike in murine macrophages, highlighting a difference between murine and human macrophages.

Overall, our findings are timely, given a recent report that showed that there is an increase in the number of choroidal macrophages in human eyes with AMD (36), strongly implicating a pathogenic role for macrophages in disease. Moreover, our findings may partially explain why activated macrophages derived from neovascular AMD patients have proangiogenic characteristics $(37,38)$. While other groups have suggested that endothelial miR-150 may suppress pathologic ocular neovascularization $(39,40)$, our findings highlight a distinct, macrophage-specific role for miR-150 that, in fact, promotes pathological ocular neovascularization. These differences are not surprising, given that miRs can act in a cell-specific manner. Ultimately, these findings open up therapeutic vistas for miR-based therapies for AMD. Moreover, understanding the mechanisms that cause macrophage aging and how such aging contributes to AMD also has broad applicability to other age-associated diseases by educating us about critical unifying pathways that drive their pathobiology.

\section{Methods}

Animals. We obtained 18-month-old, WT C57BL/6J mice from the National Institute on Aging and compared them with strain-matched, young (i.e., 2- to 3-month-old) WT controls. We obtained mice with floxed Fasn alleles $\left(F_{a s n^{\mathrm{f} / \mathrm{f}}}\right)(41)$ from Clay Semenkovich (Washington University School of Medicine) and mice with floxed $S c d 2$ alleles $\left(S c d 2^{\mathrm{f} / \mathrm{f}}\right)(42,43)$ from Hide Tsukamoto (Keck School of Medicine of the University of Southern California, Los Angeles, California, USA). We crossed these floxed mice with mice carrying the lysozyme M-Cre (LysMcre) transgene (44) to generate mice with myelomonocytic-specific deletion of Fasn $\left(\right.$ Fasn $\left.^{-\mathrm{m} / \mathrm{m}}\right)$ and $S c d 2\left(S c d 2^{-\mathrm{m} / \mathrm{m}}\right)$. We harvested PMs, SMs, and BMDMs from female mice at the ages indicated and used equal proportions of male and female mice for laser-injury CNV experiments. Unless otherwise specified, we used mice that were 2-3 months of age for experimentation.

Macrophages. We harvested PMs from mice 5 days after elicitation with $4 \%$ thioglycollate (MilliporeSig$\mathrm{ma}$ ). We harvested SMs from mice by mincing spleens with a razor blade, incubating in spleen dissociation medium (Stemcell Technologies) for 30 minutes at room temperature, and performing magnetic cell separation with the PE selection kit (Stemcell Technologies) and PE-conjugated F4/80 monoclonal antibody (clone BM8; eBioscience), following manufacturer's instructions. We cultured PMs and SMs in RPMI 1640 medium (Thermo Fisher Scientific) supplemented with 10\% FBS (Atlanta Biologicals) and 1\% penicillin-streptomycin (Thermo Fisher Scientific). Additionally, we generated BMDMs by culturing BM aspirates from mice in RPMI 1640 medium (Thermo Fisher Scientific) supplemented with 20\% L929-conditioned medium, 10\% FBS (Atlanta Biologicals), 1\% GlutaMAX (Thermo Fisher Scientific), and 1\% penicillin-streptomycin (Thermo Fisher Scientific). Monocytes were allowed to differentiate for 1 week prior to experimentation. When indicated, we treated macrophages with $25 \mu \mathrm{g} / \mathrm{ml}$ oxLDL (Intracel) for 24 hours, $25 \mu \mathrm{g} / \mathrm{ml}$ acLDL (Intracel) for 24 hours, or $100 \mathrm{ng} / \mathrm{ml}$ LPS (MilliporeSigma) for 24 hours.

$m i R$ microarray. We profiled miR expression of young and aged PMs that (i) were left untreated, (ii) were treated with $25 \mu \mathrm{g} / \mathrm{ml}$ acLDL for 24 hours, or (iii) were treated with $25 \mu \mathrm{g} / \mathrm{ml}$ oxLDL for 24 hours (total of 6 groups; 3 treatments $\times 2$ ages). We extracted RNA with the mirVana miRNA isolation kit (Thermo Fisher Scientific), determining quantity and quality of the RNA with a 2100 BioAnalyzer and the Total RNA Pico kit (Agilent Technologies). All samples $(n=24)$ had RNA integrity numbers $(\mathrm{RIN})>9.5$. We labeled each sample with FlashTag Biotin HSR RNA Labeling Kits (Affymetrix) to prepare them for the GeneChip miRNA 3.0 Array. We processed the array results with Affymetrix Expression Console (v1.3.1.187) at standard settings 
(RMA background correction, median polish summarization, and quantile normalization) to generate intensity values with a second set of data produced without quantile normalization. We filtered the data by probeset type and by detection call and removed probesets without a "detected" call in any of the 24 samples; after this filtering, we retained 1,093 of the initial 1,966 probesets for further statistical analysis. To identify potential outliers, we performed principal component analysis (PCA) and hierarchical clustering and assessed quality control (QC) metrics from Expression Console (e.g., all probeset RLE means > 0.25). This analysis identified 3 outliers, which we omitted from further analysis. We performed statistical analysis with the R package "limma" (45) to generate lists of miRs differentially expressed in our various groups based on their $P$ values and FDR-adjusted $P$ values (i.e., $q$ values). The microarray data are available at the Gene Expression Omnibus (GEO) at NCBI under accession number GSE111323.

miR expression profiling. For miR expression profiling, we extracted total RNA with the mirVana miRNA isolation kit (Thermo Fisher Scientific) and prepared cDNA with the universal cDNA synthesis kit II (Exiqon). We then performed qPCR using ExiLENT SYBR Green master mix (Exiqon) and miR LNA primer sets (Exiqon). To analyze the data, we used the $\Delta \Delta \mathrm{CT}$ method, normalizing to U6 expression. A list of primers used for miR qPCR is provided in Supplemental Table 2.

$m R N A$ expression profiling. For mRNA expression profiling, we extracted RNA with the RNeasy kit (Qiagen) and prepared cDNA with the high-capacity cDNA reverse transcription kit (Thermo Fisher Scientific). We then performed qPCR with TaqMan fast advanced master mix (Thermo Fisher Scientific) and TaqMan real-time PCR gene expression assays (Thermo Fisher Scientific). A list of primers used for qPCR is provided in Supplemental Table 2. We also profiled mRNA expression with custom TaqMan array plates (Thermo Fisher Scientific) with the assays indicated in Supplemental Table 3. We used the $\Delta \Delta C T$ method, normalizing to 1 or more housekeeping gene, as appropriate.

RNA-Seq. We extracted total RNA from PMs transfected with either synthetic miR-150-5p or NC (Exiqon) with TRIzol (Thermo Fisher Scientific), followed by column purification with the RNeasy Plus mini kit (Qiagen). We determined the quantity and quality of the RNA samples with a 2100 BioAnalyzer and the Total RNA Pico kit (Agilent Technologies). All samples $(n=12)$ had RIN > 9.6. We analyzed the transcriptomes of PMs after miR-150 overexpression with RNA-Seq, with an initial input of $600 \mathrm{ng}$ of total RNA per sample before mRNA enrichment with the rRNA Ribo-Zero rRNA removal kit (Illumina). We prepared sequencing libraries with standard protocols. QC revealed improper fragmentation of $1 \mathrm{sam}-$ ple, which was omitted from further analysis. The remaining samples $(n=11)$ were sequenced in 2 flowcell lanes on a HiSeq 2500 (Illumina) at the Washington University Genome Technology Access Center (GTAC). We mapped the sequencing reads to the genome with Spliced Transcripts Alignment to a Reference (STAR) (https://github.com/alexdobin/STAR). Next, we performed a standard EdgeR and Sailfish analysis of gene-level features. We defined a significant up- or downregulation as a |fold-change| $>1.20$ with a FDR $<0.20$. We performed pathway analysis for enrichment in GO processes, process networks, and pathway maps with MetaCore (Clarivate Analytics). The RNA-Seq data are available at the GEO at NCBI under accession number GSE111323.

Lipidomic analysis. We suspended macrophages in PBS at $2.0 \times 10^{6}$ cells $/ \mathrm{ml}$ and performed protein precipitation from $100 \mu 1$ of macrophage suspension to extract ceramides, PCs, PEs, PIs, PGs, and PSs. Prior to extraction, we added deuterated $\mathrm{d}_{5}$-Cer (16:0), $\mathrm{d}_{4}$-Cer (22:0), and $\mathrm{d}_{4}$-Cer (24:0) as internal standards for ceramides and PC (28:2), PE (32:2), PG (30:0), PI (32:0), and PS (28:0) as internal standards for the other lipid classes. We measured lipids with a Shimadzu 10A HPLC system and a Shimadzu SIL-20AC HT auto-sampler coupled to a Thermo Scientific TSQ Quantum Ultra triple quadrupole mass spectrometer operated in SRM mode under ESI(+). We conducted data processing with Xcalibur (Thermo Fisher Scientific). We prepared QC samples by pooling aliquots of the study samples and injected them between every 5 samples to monitor instrument performance, omitting lipid species with coefficients of variance $>15 \%$ in QC samples. We performed relative quantification by comparing the peak area ratios of the analytes to the corresponding internal standards.

Human subjects. To isolate PBMCs, we performed density gradient centrifugation with BD Vacutainer CPT cell preparation tubes. We stored PBMC pellets at $-80^{\circ} \mathrm{C}$ until further analysis. We classified patients as no AMD, early AMD, or wet AMD based on established clinical criteria (46). Early AMD patients had either moderate drusen $(>63 \mu \mathrm{m})$ or pigment changes in at least 1 eye but no CNV or GA in either eye at the time of sample collection. Wet AMD patients had CNV in at least 1 eye at the time of sample collection. We excluded patients with pattern dystrophy, macular telangiectasia, dominant drusen, or central serous chorioretinopathy. 
Absolute miR-150 quantification in human PBMCs. We extracted RNA from PBMCs with the mirVana miRNA isolation kit (Thermo Fisher Scientific) and performed reverse transcription (RT) with the universal cDNA synthesis kit II (Exiqon) by adding $2 \mu 1$ of RNA to $2 \mu 1$ of $5 \times$ reaction buffer, $1 \mu$ l of enzyme mix, and $5 \mu \mathrm{l}$ of nuclease-free $\mathrm{H}_{2} \mathrm{O}$ (total volume, $10 \mu \mathrm{l}$ ). We diluted the cDNA to a final volume of $60 \mu \mathrm{l}$ and performed miRCURY LNA Universal RT miR PCR by adding $4 \mu 1$ of diluted cDNA to $1 \mu 1$ of the appropriate primer and $5 \mu$ of ExiLENT SYBR Green master mix (total volume, $10 \mu 1$ ). To determine absolute copy number of human-miR-150-5p (hsa-miR-150-5p), we prepared standard curves with serial dilutions of synthetic hsa-miR-150-5p (Integrated DNA Technologies) ranging from $1 \times 10^{4}$ copies per 2 $\mu 1$ input to $1 \times 10^{10}$ copies per $2 \mu 1$ input, followed by RT and PCR as above. To account for differences in RNA extraction and RT efficiency performed on separate occasions, we spiked in 20 fmol of synthetic C. elegans-miR-39-3p (cel-miR-39-3p) (Integrated DNA Technologies) into each sample prior to RNA extraction and normalized to the global arithmetic mean. We normalized miR-150 copy number for each patient by dividing by the total RNA used for RT.

miR-150 overexpression. To transiently overexpress miR-150 in PMs, we used commercially available miRCURY LNA miR mimics (Exiqon), following standard protocols for fast-forward transfection. In short, we plated PMs at $1 \times 10^{6}$ cells/well in 6-well plates. Two hours after plating, we prepared transfection complexes by combining miR mimic $(20 \mathrm{nM})$ and HiPerFect transfection reagent (18 $\mu 1 /$ well; Qiagen) in RPMI medium supplemented with 10\% FBS (Atlanta Biologicals) and 1\% penicillin-streptomycin (Thermo Fisher Scientific), incubating for 15 minutes at room temperature before adding to the cells. We assessed transfection efficiency with fluorescence microscopy by visualizing fluorescein amidite-labeled (FAM-labeled) miR mimics.

miR-150 target identification. To identify miR-150 targets, we retrieved 3' UTR sequences from the UCSC Genome Brower (genome.ucsc.edu; Mouse Assembly GRCm38/mm10) and manually searched for the presence of relevant miR-150 seed sequences (8mer site: 3' - UUGGGAGA - 5'; 7mer-m8 site: 3' - UUGGGAG - 5'; 7mer-A1 site: 3' - UGGGAGA - 5'; 6mer site: 3' - UGGGAG - 5'; offset 6mer site: 3' - UUGGGA - 5'). Additionally, we used existing miR target identification databases: TargetScanMouse (release 7.1) (47), DIANA-microT-CDS (version 5.0) (48), DIANA-TarBase (version 7.0) (49), miRDB (50), RNA22 (version 2.0) (51), and microRNA.org (August 2010 release) (52).

Fasn/Scd2 knockdown. To transiently knock down Fasn and Scd2, we used commercially available FlexiTube siRNA (Qiagen), following standard protocols for fast-forward transfection. In short, we plated PMs at $1 \times 10^{6}$ cells/well in 6-well plates. Two hours after plating, we prepared siRNA transfection complexes by combining siRNA ( $50 \mathrm{nM})$ and HiPerFect transfection reagent (18 $\mu$ l/well; Qiagen) in RPMI medium supplemented with 10\% FBS (Atlanta Biologicals) and 1\% penicillin-streptomycin (Thermo Fisher Scientific), incubating for 15 minutes at room temperature before adding to the cells. We confirmed efficient siRNA knockdown by qPCR. We analyzed macrophage activation and function 48 hours after transfection.

miRNA target dual-reporter assays. We ordered custom miTarget 3' UTR miRNA target clones from GeneCopoeia with WT or mutated $S c d 23^{\prime}$ UTR inserted downstream of a secreted GLuc reporter gene driven by the SV40 promoter and a secreted alkaline phosphatase (SEAP) reporter gene driven by a CMV promoter. We cotransfected 293T cells (ATCC), cultured routinely in high-glucose DMEM (Thermo Fisher Scientific) supplemented with 10\% FBS (Atlanta Biologicals), with the target clone and either miR-150 mimic or nontargeting NC mimic (Exiqon), following manufacturer's instructions. In brief, we plated 293T cells in 96-well plates at $2 \times 10^{4}$ cells/well the day before transfection. On the day of transfection, we prepared transfection complexes by combining the target clone (100 ng/well), miR mimic (20 nM), Lipofectamine 3000 (0.15 $\mu \mathrm{l} /$ well; Thermo Fisher Scientific), and P3000 reagent (0.20 $\mu \mathrm{l} /$ well) in Opti-MEM medium (Thermo Fisher Scientific) and incubating for 30 minutes at room temperature before adding to the cells. Forty-eight hours after transfection, we collected the supernatant to measure GLuc activity with the Secrete Pair Dual Luminescence assay kit (GeneCopoeia). We normalized for transfection efficiency by measuring SEAP activity. We optimized cotransfection conditions with a positive-control pmaxGFP plasmid (Lonza).

CNV experiments. We performed laser-induced $\mathrm{CNV}$ as described previously (53). Briefly, we anesthetized mice and placed 4 laser spots around the optic disc ( $200 \mathrm{~mW}, 0.1$ second, $100 \mu \mathrm{m}$ spot size) using a slit-lamp delivery system with a cover glass as a contact lens. Seven days after injury, we perfused the mice with 2,000 $\mu 1$ of $5 \mathrm{mg} / \mathrm{ml}$ FITC-dextran (molecular weight, 2,000,000 daltons; MilliporeSigma) through the left ventricle. We then enucleated the eyes and fixed them in 2\% paraformaldehyde (PFA; Alfa Aesar) for 30 minutes. After fixation, we washed the eyes with PBS and flat-mounted the retinal pigment epithelium-choroid (RPE-choroid) 
complex onto a glass slide. We acquired Z-stack images of the CNV spots using an Olympus FV1000 confocal microscope and processed images with ImageJ $(\mathrm{NIH})$ to generate pseudo-volumetric 2-dimensional images. After excluding laser spots that resulted in retinal, subretinal, or vitreous hemorrhage, we quantified pixel intensity using MetaMorph (Molecular Devices). For immunotherapy experiments, we adoptively transferred 100,000 macrophages into each eye in $2 \mu \mathrm{PBS}$ immediately following laser injury via intravitreal injection with a $10 \mu \mathrm{l}$ Neuros Model $1701 \mathrm{RN}$ syringe (point style 4) and small-hub, 31-gauge needles (Hamilton).

VEGF measurements. We measured VEGF secretion in supernatants from macrophages with the mouse VEGF Quantikine ELISA kit (R\&D Systems), following manufacturer's instructions.

Statistics. We performed statistical analysis with Prism 5 (Graphpad) or SPSS Statistics (Version 23; IBM). We assessed the normality of our data graphically and with the Kolmogorov-Smirnov test, using nonparametric alternatives when appropriate. When comparing a single variable between 2 groups, we used 2 -tailed $t$ tests or 2-tailed Mann-Whitney $U$ tests. For other analyses, we performed the appropriate statistical test for each type of data, as indicated in figure legends. A $P$ value less than 0.05 was considered significant. Open circles depict individual data points or individual human subjects. Box-and-whisker plots depict medians, with boxes outlining the interquartile range and whiskers indicating the minima and maxima.

We performed an a priori power calculation with $\mathrm{G}^{*}$ Power 3.1 (54) to determine the appropriate sample size for our human studies. To detect a significant difference between AMD patients and control subjects (allocation ratio: 1.0:1.5) at the 2-sided $\alpha=0.05$ level with an estimated effect size $d$ of 0.6 based on pilot experiments and $80 \%$ power, we calculated that we needed to recruit 94 subjects total. We exceeded this threshold $(n=106)$, yielding adequate statistical power to detect our anticipated difference. To model the relationship between PBMC miR-150 levels and AMD, we generated a binary logistic regression model. Our model included the predictor variables of age at the time of sample collection, sex, $\operatorname{and} \log _{10}$-transformed PBMC miR-150 levels. We assessed model fit with the Hosmer-Lemeshow lack-of-fit test and performed model diagnostics by examining Cook's distances, leverages, and residual deviances. No cases were omitted upon sensitivity analysis. We checked for collinearity by examining variance inflation factors (VIF). We used an unadjusted $\alpha$ of 0.05 for the binary logistic regression.

Study approval. All animal experiments were reviewed and approved by the IACUC of Washington University in St. Louis and performed in accordance with the Washington University School of Medicine Animal Care and Use guidelines. The human study was reviewed and approved by the Human Research Protection Office of Washington University in St. Louis and adhered to the Declaration of Helsinki. We obtained informed consent from all human subjects prior to blood collection.

\section{Author contributions}

Conceptualization was contributed by RSA, Jonathan B. Lin, and AS. Investigation was contributed by Jonathan B. Lin, HVM, AS, RS, PK, Joseph B. Lin, ZD, and NB. Writing of the original draft was contributed by Jonathan B. Lin. Review and editing was contributed by Jonathan B. Lin, RSA, DSO, AS, HVM, and Joseph B. Lin. Supervision was contributed by RSA and DSO. Funding acquisition was contributed by RSA, DSO, and Jonathan B. Lin.

\section{Acknowledgments}

This work was supported by NIH grants R01 EY019287 (RSA), P30 EY02687 (Vision Core Grant), P30 DK020579 (Diabetes Research Center Metabolomics Core), UL1 TR000448 (GTAC), and P30 CA91842 (GTAC); the Starr Foundation (RSA); the Carl Marshall Reeves and Mildred Almen Reeves Foundation (RSA); the Bill and Emily Kuzma Family Gift for retinal research (RSA); a Physician-Scientist Award and a Nelson Trust Award from Research to Prevent Blindness (RSA); the Jeffrey Fort Innovation Fund (RSA); the Glenn Foundation (RSA); and the Thome Foundation (RSA). Additional funding comes from an unrestricted grant to the Department of Ophthalmology and Visual Sciences of Washington University School of Medicine from Research to Prevent Blindness. JBL was supported by the Washington University in St. Louis Medical Scientist Training Program (NIH grant T32 GM07200), the Washington University in St. Louis Institute of Clinical and Translational Sciences (NIH grants UL1 TR002345, TL1 TR002344), and the VitreoRetinal Surgery Foundation. The authors thank Andrea Santeford, Alexander Cammack, Howard Chen, Michael Casey, Rei Nakamura, and Nicole Zapata for technical assistance; Clay Semenkovich and Hide Tsukamoto for providing mutant mice; and Danyel Cavazos for help with scientific illustration. 
Address correspondence to: Rajendra S. Apte, 660 South Euclid Avenue, Box 8096, St. Louis, Missouri 63110, USA. Phone: 314.362.3315; Email: apte@wustl.edu.

1. Khalil H, et al. Aging is associated with hypermethylation of autophagy genes in macrophages. Epigenetics. 2016;11(5):381-388

2. Wong CK, Smith CA, Sakamoto K, Kaminski N, Koff JL, Goldstein DR. Aging Impairs Alveolar Macrophage Phagocytosis and Increases Influenza-Induced Mortality in Mice. J Immunol. 2017;199(3):1060-1068.

3. Kelly J, Ali Khan A, Yin J, Ferguson TA, Apte RS. Senescence regulates macrophage activation and angiogenic fate at sites of tissue injury in mice. J Clin Invest. 2007;117(11):3421-3426.

4. Sene A, et al. Impaired cholesterol efflux in senescent macrophages promotes age-related macular degeneration. Cell Metab. 2013;17(4):549-561.

5. Sene A, Apte RS. Eyeballing cholesterol efflux and macrophage function in disease pathogenesis. Trends Endocrinol Metab. 2014;25(3):107-114.

6. Ma W, Wong WT. Aging Changes in Retinal Microglia and their Relevance to Age-related Retinal Disease. Adv Exp Med Biol. 2016;854:73-78

7. van Leeuwen R, Klaver CC, Vingerling JR, Hofman A, de Jong PT. Epidemiology of age-related maculopathy: a review. Eur J Epidemiol. 2003;18(9):845-854.

8. Sene A, Chin-Yee D, Apte RS. Seeing through VEGF: innate and adaptive immunity in pathological angiogenesis in the eye Trends Mol Med. 2015;21(1):43-51.

9. Ferris FL, Fine SL, Hyman L. Age-related macular degeneration and blindness due to neovascular maculopathy. Arch Ophthalmol. 1984;102(11):1640-1642.

10. Neale BM, et al. Genome-wide association study of advanced age-related macular degeneration identifies a role of the hepatic lipase gene (LIPC). Proc Natl Acad Sci USA. 2010;107(16):7395-7400.

11. Gerrity RG. The role of the monocyte in atherogenesis: I. Transition of blood-borne monocytes into foam cells in fatty lesions. Am J Pathol. 1981;103(2):181-190.

12. Gerrity RG, Naito HK, Richardson M, Schwartz CJ. Dietary induced atherogenesis in swine. Morphology of the intima in prelesion stages. Am J Pathol. 1979;95(3):775-792.

13. Chinetti-Gbaguidi G, Colin S, Staels B. Macrophage subsets in atherosclerosis. Nat Rev Cardiol. 2015;12(1):10-17.

14. Peled M, Fisher EA. Dynamic Aspects of Macrophage Polarization during Atherosclerosis Progression and Regression. Front Immunol. 2014;5:579.

15. Fadini GP, et al. Pro-inflammatory monocyte-macrophage polarization imbalance in human hypercholesterolemia and atherosclerosis. Atherosclerosis. 2014;237(2):805-808.

16. Ishikawa T, Ayaori M, Uto-Kondo H, Nakajima T, Mutoh M, Ikewaki K. High-density lipoprotein cholesterol efflux capacity as a relevant predictor of atherosclerotic coronary disease. Atherosclerosis. 2015;242(1):318-322.

17. Crabb JW, et al. Drusen proteome analysis: an approach to the etiology of age-related macular degeneration. Proc Natl Acad Sci USA. 2002;99(23):14682-14687.

18. Curcio CA, Johnson M, Huang JD, Rudolf M. Aging, age-related macular degeneration, and the response-to-retention of apolipoprotein B-containing lipoproteins. Prog Retin Eye Res. 2009;28(6):393-422.

19. Kramsch DM, Franzblau C, Hollander W. The protein and lipid composition of arterial elastin and its relationship to lipid accumulation in the atherosclerotic plaque. J Clin Invest. 1971;50(8):1666-1677.

20. Mullins RF, Russell SR, Anderson DH, Hageman GS. Drusen associated with aging and age-related macular degeneration contain proteins common to extracellular deposits associated with atherosclerosis, elastosis, amyloidosis, and dense deposit disease. FASEB J. 2000;14(7):835-846.

21. Vavvas DG, et al. Regression of Some High-risk Features of Age-related Macular Degeneration (AMD) in Patients Receiving Intensive Statin Treatment. EBioMedicine. 2016;5:198-203.

22. Apte RS. Targeting Tissue Lipids in Age-related Macular Degeneration. EBioMedicine. 2016;5:26-27.

23. Gehlbach P, Li T, Hatef E. Statins for age-related macular degeneration. Cochrane Database Syst Rev. 2016;(8):Cd006927.

24. Grassmann F, et al. A circulating microrna profile is associated with late-stage neovascular age-related macular degeneration. PLoS One. 2014;9(9):e107461.

25. Ménard C, et al. MicroRNA signatures in vitreous humour and plasma of patients with exudative AMD. Oncotarget. 2016;7(15):19171-19184.

26. Wei X, et al. Fatty acid synthesis configures the plasma membrane for inflammation in diabetes. Nature. 2016;539(7628):294-298.

27. Apte RS, Richter J, Herndon J, Ferguson TA. Macrophages inhibit neovascularization in a murine model of age-related macular degeneration. PLoS Med. 2006;3(8):e310.

28. Xiao C, et al. MiR-150 controls B cell differentiation by targeting the transcription factor c-Myb. Cell. 2007;131(1):146-159.

29. Heinz RE, et al. Constitutive expression of microRNA-150 in mammary epithelium suppresses secretory activation and impairs de novo lipogenesis. Development. 2016;143(22):4236-4248.

30. Rayner KJ, et al. Antagonism of miR-33 in mice promotes reverse cholesterol transport and regression of atherosclerosis. $J$ Clin Invest. 2011;121(7):2921-2931.

31. Meiler S, Baumer Y, Toulmin E, Seng K, Boisvert WA. MicroRNA 302a is a novel modulator of cholesterol homeostasis and atherosclerosis. Arterioscler Thromb Vasc Biol. 2015;35(2):323-331.

32. Lv YC, et al. MicroRNA-19b promotes macrophage cholesterol accumulation and aortic atherosclerosis by targeting ATP-binding cassette transporter A1. Atherosclerosis. 2014;236(1):215-226.

33. Schilling JD, et al. Palmitate and lipopolysaccharide trigger synergistic ceramide production in primary macrophages. $J$ Biol Chem. 2013;288(5):2923-2932.

34. Tarasov K, et al. Molecular lipids identify cardiovascular risk and are efficiently lowered by simvastatin and PCSK9 deficiency. J Clin Endocrinol Metab. 2014;99(1):E45-E52. 
35. Miyazaki M, Dobrzyn A, Elias PM, Ntambi JM. Stearoyl-CoA desaturase-2 gene expression is required for lipid synthesis during early skin and liver development. Proc Natl Acad Sci USA. 2005;102(35):12501-12506.

36. McLeod DS, Bhutto I, Edwards MM, Silver RE, Seddon JM, Lutty GA. Distribution and Quantification of Choroidal Macrophages in Human Eyes With Age-Related Macular Degeneration. Invest Ophthalmol Vis Sci. 2016;57(14):5843-5855.

37. Hagbi-Levi S, et al. Proangiogenic characteristics of activated macrophages from patients with age-related macular degeneration. Neurobiol Aging. 2017;51:71-82.

38. Nakamura R, et al. IL10-driven STAT3 signalling in senescent macrophages promotes pathological eye angiogenesis. Nat Commun. 2015;6:7847.

39. Liu CH, et al. Endothelial microRNA-150 is an intrinsic suppressor of pathologic ocular neovascularization. Proc Natl Acad Sci USA. 2015;112(39):12163-12168.

40. Shen J, et al. MicroRNAs regulate ocular neovascularization. Mol Ther. 2008;16(7):1208-1216.

41. Chakravarthy MV, et al. "New" hepatic fat activates PPARalpha to maintain glucose, lipid, and cholesterol homeostasis. Cell Metab. 2005;1(5):309-322.

42. Lai KKY, et al. Stearoyl-CoA Desaturase Promotes Liver Fibrosis and Tumor Development in Mice via a Wnt Positive-Signaling Loop by Stabilization of Low-Density Lipoprotein-Receptor-Related Proteins 5 and 6. Gastroenterology. 2017;152(6):1477-1491.

43. Masuda M, et al. Saturated phosphatidic acids mediate saturated fatty acid-induced vascular calcification and lipotoxicity. $J C l i n$ Invest. 2015;125(12):4544-4558.

44. Clausen BE, Burkhardt C, Reith W, Renkawitz R, Förster I. Conditional gene targeting in macrophages and granulocytes using LysMcre mice. Transgenic Res. 1999;8(4):265-277.

45. Ritchie ME, et al. limma powers differential expression analyses for RNA-sequencing and microarray studies. Nucleic Acids Res. 2015;43(7):e47

46. Ferris FL, et al. A simplified severity scale for age-related macular degeneration: AREDS Report No. 18. Arch Ophthalmol. 2005;123(11):1570-1574.

47. Agarwal V, Bell GW, Nam JW, Bartel DP. Predicting effective microRNA target sites in mammalian mRNAs. Elife. $2015 ; 4$.

48. Paraskevopoulou MD, et al. DIANA-microT web server v5.0: service integration into miRNA functional analysis workflows. Nucleic Acids Res. 2013;41(Web Server issue):W169-W173.

49. Vlachos IS, et al. DIANA-TarBase v7.0: indexing more than half a million experimentally supported miRNA:mRNA interactions. Nucleic Acids Res. 2015;43(Database issue):D153-D159.

50. Wong N, Wang X. miRDB: an online resource for microRNA target prediction and functional annotations. Nucleic Acids Res. 2015;43(Database issue):D146-D152.

51. Miranda KC, et al. A pattern-based method for the identification of MicroRNA binding sites and their corresponding heteroduplexes. Cell. 2006;126(6):1203-1217.

52. Betel D, Wilson M, Gabow A, Marks DS, Sander C. The microRNA.org resource: targets and expression. Nucleic Acids Res. 2008;36(Database issue):D149-D153.

53. Dong Z, et al. Specific inhibition of serine/arginine-rich protein kinase attenuates choroidal neovascularization. Mol Vis. 2013;19:536-543

54. Faul F, Erdfelder E, Lang AG, Buchner A. G*Power 3: a flexible statistical power analysis program for the social, behavioral, and biomedical sciences. Behav Res Methods. 2007;39(2):175-191. 